\title{
Floristic study of Aphaedo Island in Shinan-gun, Jeollanam-do, Korea
}

\section{Jin-Oh HYUN*, Hye Ryun NA, Yeonsu KIM and Byungwoo HAN}

\author{
Northeastern Asia Biodiversity Institute, Seoul 05677, Korea \\ (Received 18 January 2018; Revised 6 February 2018; Accepted 13 March 2018)
}

\begin{abstract}
We investigated vascular plants of Aphaedo Island in Shinan-gun, Jeollanam-do, Korea. By referring to voucher specimens collected over the course of 28 days from May of 2011 to March of 2016, a total of 451 taxa were identified and grouped into 102 families, 294 genera, 413 species, 6 subspecies, 30 varieties, and 2 forms, of which 9 taxa were classified as endangered or rare, including Albizia kalkora, Salomonia oblongifolia, and Centranthera cochinchinensis var. lutea. A total of 59 taxa were identified as regional indicator plants. Six taxa were endemic to Korea, including Hepatica insularis, Indigofera koreana, and Lespedeza maritima. Three taxa (Rumex acetosella, Aster pilosus, and Hypochaeris radicata) among 52 naturalized taxa were ecosystem-disturbing plants as designated by the Ministry of the Environment. The results of preceding floristic research before and after the inauguration of the Aphaedaegyo (bridge) were used to analyze changes in the number of naturalized species on Aphaedo Island.
\end{abstract}

Keywords: Aphaedo Island, flora, endangered and rare plants, floristic regional indicator plants, endemic plants, naturalized plants

Aphaedo Island is located in the southwest end of Korea. It belongs to Aphae-eup in Shinan-gun in Jeollanam-do. Aphaedo Island is adjacent to Muan-gun on the east, Amtaedo Island on the west, Mokpo-si on the south, and Jido Island on the north. Aphaedo Island is connected to Mokpo-si through the Aphaedaegyo Bridge. It is also connected with Muan-gun through the Kimdaejungdaegyo Bridge. Aphaedo Island is the main island of Aphae-eup consisting of 60 uninhabited islands and 8 inhabited islands, including Garando Island, Goyido Island, and Maehwado Island. The area of Aphaedo Island is $48.87 \mathrm{~km}^{2}$, and the coastline reaches $81.9 \mathrm{~km}$ (Shinan-gun Office, 2016). Aphaedo Island is a mountainous area formed by Holmaesan Mountain (or Inmaesan Mountain, elev. 138.2 $\mathrm{m})$ to the north of the island and Songgongsan Mountain (elev. $234.1 \mathrm{~m}$ ) which is the highest peak of the western end. The center and the southeast of the island are flat while its outskirts are hilly areas of about $100 \mathrm{~m}$ in elevation. The entire island is surrounded by tidal-flats. Its coastline has a lot of bays and capes. However, its coastline is monotonous due to reclamation and paddy fields. It is said that the name (Aphae, 押海 in Chinese character) of this island is derived from the shape of the island spreading on three sides, pressing (押) the sea (海) (National Geographic Information Institute, 2010).

Shinan-gun, including Aphaedo Island, has an average annual temperature of $14.4^{\circ} \mathrm{C}$ as of 2015 . Its average temperatures in January (the coldest month) and in August (the hottest month) are $2.5^{\circ} \mathrm{C}$ and $25.9^{\circ} \mathrm{C}$, respectively, with a difference of $23.4^{\circ} \mathrm{C}$. It has a temperate oceanic climate (Shinan-gun Office, 2016). Aphaedo Island belongs to the south coast subdistrict of the floristic region of the Korean peninsula (Lee and Yim, 2002). A total of 154 taxa (134 species, 18 varieties, and 2 forms) of 61 families and 124 genera were recorded at Aphaedo Island in floristic studies for 32 islands (distributed in the West and South Coast of Korea) conducted from June 2003 to October 2003 by Park (2004). Jeong et al. (2011) have reported 103 taxa from Aphaedo Island based on voucher specimens obtained from 11 islands in Shinan-gun in 2003 and 2010 (the list of plants growing in the region was counted as 102 taxa). Nam et al. (2012) have surveyed the flora of three islands (Aphaedo Island, Bigeumdo Island, Dochodo Island) belonging to Shinan-gun from May to September 2010 and reported 379

\footnotetext{
*Author for correspondence: megalamen@daum.net
} 
taxa from Aphaedo Island (the list of plants growing in the region was counted as 380 taxa). Hwang et al. (2013) have surveyed 15 islands in Shinan-gun from September 2004 to September 2010 and reported 287 taxa from Aphaedo Island. Kim (2008) has also reported 68 taxa of naturalized plants from Aphaedo Island in an ecological study on vegetation distribution of coastal islands.

The objective of the present study was to update vascular plant flora of Aphaedo Island and determine the distribution of major plants such as endangered species, rare plants, endemic plants of the Korean Peninsula, and regional indicator species representing the south coast subdistrict flora. Another purpose of this study was to understand changes of flora in comparison with previous studies. The island was connected to the main land by Aphaedaegyo in 2008. After that, Shinangun Office was moved in 2011 and Kimdaejungdaegyo was opened in 2013. In 2018 there will be an inauguration of the Saecheonnyeondaegyo Bridge connecting Aphaedo Island and Amtaedo Island. This study was conducted to obtain basic data on the conservation and management of Aphaedo Island's biological resources, which are under high development pressure as the island is Shinan-gun's administrative and transportation center.

\section{Materials and Methods}

A field survey was conducted for a total of 28 days from May 2011 to March 2016 to examine the flora of Aphaedo Island. The scope of the survey covered vascular plants that appeared all over the area of Aphaedo Island, encompassing 10 administrative districts: Songgong-ri and Daecheon-ri, including Songgongsan Mountain, the highest peak (234.1 m) in the island; Dongseo-ri and Bunmae-ri (plains district through which Route 2 passes); Sinsang-ri (where Shinan-gun Office and Aphaedaegyo is located); Janggam-ri; Hakgyo-ri (including town office); Bongryong-ri (with Kimdaejungdaegyo connecting to mainland); Garyong-ri and Sinyong-ri (composed of hilly areas and farmlands of Holmaesan Mountain) (Fig. 1, Table 1). We collected samples in various plant growth environments such as coast, mountains, villages, farmland, and roads in the survey area on foot. In this process, individuals with reproductive organs such as flower, fruit, and sporangium were collected and prepared as dried specimens. Location, habitat, and identification of specimens were entered into the National Biological Resources Input System of the National Institute of Biological Resources (NIBR) in Korea. Voucher specimens were deposited at the herbarium of NIBR (KB).

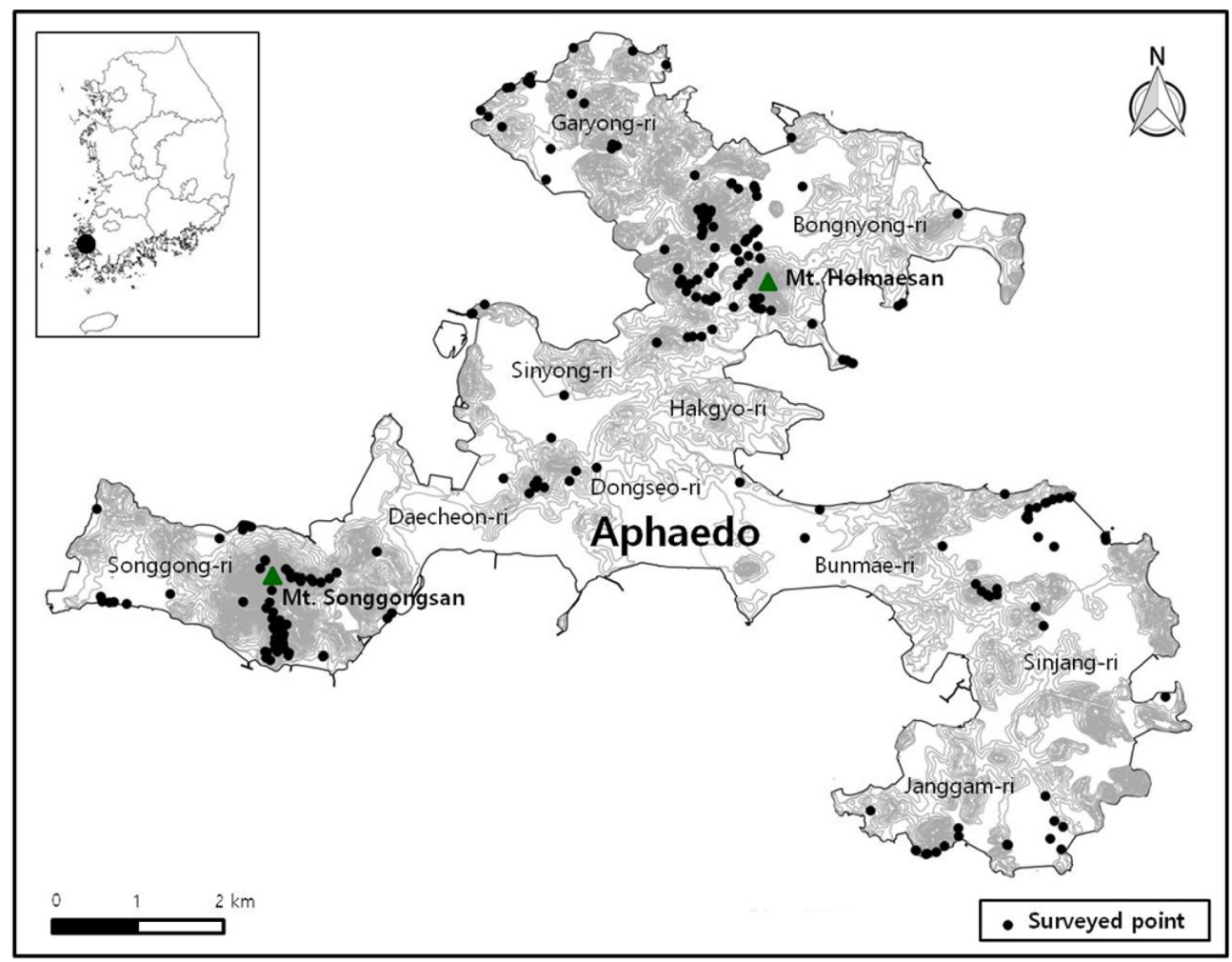

Fig. 1. Map of the investigated area (Aphaedo Island). 
Identification and classification of these collected plants were performed with reference to Lee (1996a, 1996b), Lee (2003), Lee (2006), Park (2009), Park et al. (2008, 2011), and Hoshino and Masaki (2011). Scientific and common names were based on National List of Species of Korea (National Institute of Biological Resources, 2017) and Synonymic List of Vascular Plants in Korea (Korea National Arboretum and The Plant Taxonomic Society of Korea, 2007). The list of identified plants was arranged according to the Genera of Vascular Plants of

Table 1. Investigation timeline and location in Aphaedo Island.

\begin{tabular}{|c|c|}
\hline Date & Locality in Aphaedo Island \\
\hline 30 May 2011 & $\begin{array}{l}\text { Garyong-ri, Songgong-ri, Daecheon-ri, } \\
\text { Bunmae-ri, Sinjang-ri, Hakgyo-ri }\end{array}$ \\
\hline 29 Aug 2011 & Songgong-ri, Daecheon-ri, Sinjang-ri \\
\hline 5-6 May 2012 & $\begin{array}{l}\text { Garyong-ri, Bongnyong-ri, Bunmae-ri, } \\
\text { Hakgyo-ri, Songgong-ri, Daecheon-ri, } \\
\text { Janggam-ri }\end{array}$ \\
\hline 23-24 May 2012 & $\begin{array}{l}\text { Garyong-ri, Songgong-ri, Daecheon-ri, } \\
\text { Bongnyong-ri }\end{array}$ \\
\hline 9 Jul 2012 & Bongnyong-ri, Sinyong-ri, Garyong-ri \\
\hline 20 Aug 2012 & $\begin{array}{l}\text { Janggam-ri, Sinyong-ri, Dongseo-ri, } \\
\text { Sinjang-ri, Songgong-ri, Daecheon-ri, } \\
\text { Bunmae-ri, Hakgyo-ri }\end{array}$ \\
\hline 22 Aug 2012 & Songgong-ri \\
\hline 24-25 Sep 2012 & $\begin{array}{l}\text { Garyong-ri, Bongnyong-ri, Sinyong-ri, } \\
\text { Daecheon-ri, Songgong-ri, Bunmae-ri, } \\
\text { Janggam-ri }\end{array}$ \\
\hline $4-5$ Oct 2012 & $\begin{array}{l}\text { Sinjang-ri, Janggam-ri, Garyong-ri, } \\
\text { Songgong-ri, Daecheon-ri, Bunmae-ri }\end{array}$ \\
\hline 15 Mar 2013 & Songgong-ri \\
\hline 9 Jul 2013 & $\begin{array}{l}\text { Garyong-ri, Bongnyong-ri, Songgong-ri, } \\
\text { Sinyong-ri, Hakgyo-ri }\end{array}$ \\
\hline 9 Nov 2013 & Songgong-ri \\
\hline 22 May 2014 & Bunmae-ri, Songgong-ri \\
\hline 3 Jul 2014 & Songgong-ri \\
\hline 11 Aug 2014 & $\begin{array}{l}\text { Bongnyong-ri, Garyong-ri, Daecheon-ri, } \\
\text { Songgong-ri }\end{array}$ \\
\hline 14 Oct 2014 & Daecheon-ri, Songgong-ri, Sinjang-ri \\
\hline 12 Aug 2015 & Bunmae-ri \\
\hline 26-27 Aug 2015 & $\begin{array}{l}\text { Songgong-ri, Daecheon-ri, Dongseo-ri, } \\
\text { Garyong-ri }\end{array}$ \\
\hline 12 Sep 2015 & Songgong-ri \\
\hline 22-23 Sep 2015 & Garyong-ri, Bongnyong-ri \\
\hline 21 Oct 2015 & Daecheon-ri, Garyong-ri \\
\hline 21 Mar 2016 & $\begin{array}{l}\text { Bunmae-ri, Hakgyo-ri, Sinyong-ri, } \\
\text { Bongnyong-ri, Garyong-ri, Songgong-ri }\end{array}$ \\
\hline
\end{tabular}

Korea (Park, 2007). All plant names in list were based on voucher specimens. Taxa below the genus were arranged in alphabetical order. If there were several collections of voucher specimens for the same taxon, the first sampled specimen was presented. These investigated plants were reviewed in detail by the Red Data Book of Endangered Vascular Plants in Korea (National Institute of Biological Resources, 2012), Rare Plants Data Book in Korea (Korea National Arboretum, 2008), regional indicator species (National Institute of Environmental Research, 2012), endemic plants of Korean Peninsula (Chung et al., 2017), and the list of naturalized plants (Bang, 2014) presented in the Ministry of Environment's "1st Alien Species Management Plan".

\section{Results and Discussion}

\section{List of vascular plants}

A total of 451 taxa (413 species, 6 subspecies, 30 varieties, and 2 forms) in 102 families 294 genera of vascular plants growing on Aphaedo Island were identified, including 13 species of ferns (in 10 families 11 genera), 7 species of gymnosperms (in 2 families 4 genera), and 431 taxa (393 species, 6 subspecies, 30 varieties, and 2 forms) of angiosperms in 90 families 279 genera (Table 2, Appendix 1). This is equivalent to $10.1 \%$ of 4,455 taxa of the Korean Peninsula (National Institute of Biological Resources, 2017), 35.5\% of 1,271 taxa of vascular plants distributed in the southern coastal district of Korea (Oh et al., 2004), and 32.7\% of 1,379 species of vascular plants known to be distributed in southwestern islands in Korea (Oh et al., 2010). A total of 586 taxa (species and infra-specific taxa) have been reported from Aphaedo Island, including 154 taxa by Park (2004), 68 taxa by Kim (2008), 102 taxa by Jeong et al. (2011), 383 taxa by Nam et al. (2012), and 287 taxa by Hwang et al. (2013). Compared to previous studies, 119 taxa were newly identified in this study. Thus, a total of 705 taxa (635 species, 8 subspecies, 58 varieties, and 4 varieties) in 117 families 390 genera of vascular plants have been reported in Aphaedo Island so far, including

Table 2. Number of vascular plants in Aphaedo Island.

\begin{tabular}{cccccccc}
\hline \hline Taxa & Fam. & Gen. & Sp. & Subsp. & Var. & F. & Total \\
\hline Pteridophyta & 10 & 11 & 13 & - & - & - & 13 \\
Gymnospermae & 2 & 4 & 7 & - & - & - & 7 \\
Angiospermae & 90 & 279 & 393 & 6 & 30 & 2 & 431 \\
Dicotyledon & 74 & 200 & 276 & 4 & 19 & 1 & 300 \\
Monocotyledon & 16 & 79 & 117 & 2 & 11 & 1 & 131 \\
\hline \multicolumn{1}{c}{ Total } & 102 & 294 & 413 & 6 & 30 & 2 & 451 \\
\hline
\end{tabular}


the 119 taxa identified in the current study. Among them, cultivated plants were 25 taxa (species and infra-specific taxa), including Ginkgo biloba L., Castanea crenata Siebold \& Zucc. and Lagerstroemia indica L. etc.. Excluding cultivated plants reported in this study and previous studies, 680 taxa (611 species, 8 subspecies, 57 varieties, and 4 varieties) belonging to 110 families 373 genera of vascular plants are found to be native to Aphaedo Island (Appendix 1).

Among vascular plants identified in this study, evergreen broad-leaved trees were 11 taxa: Quercus salicina Blume, Camellia japonica L., C. sasanqua Thunb., Eurya japonica Thunb., Vaccinium bracteatum Thunb., Euonymus japonicus Thunb., Ilex crenata Thunb., Fatsia japonica (Thunb.) Decne. \& Planch., Hedera rhombea (Miq.) Bean, Vitex rotundifolia L. f., and Ligustrum japonicum Thunb.. Among these taxa, Camellia japonica, Hedera rhombea, and evergreen fern species Dicranopteris linearis (Burm. f.) Underw., Pteris multifida Poir., and Cyrtomium falcatum (L. f.) C. Presl. are southern plants that are expected to grow in population size and range expansion to the north due to temperature increase caused by global climate change. These are taxa that are designated and managed by the Ministry of Environment as national climate change index species (Lee et al., 2010). In addition, halophytes growing on salt fields and sea shores including mud flats, sand dunes, and rocks ( $\mathrm{Na}$ and Hyun, 2015) were classified into 41 taxa (species and infraspecific taxa) in 18 families 32 genera (Table 3). This corresponds to 9.1\% of plants distributed on Aphaedo Island. Among these, 15 taxa such as Salsola komarovii Iljin, Suaeda japonica Makino, and Polygonum fusco-ochreatum Kom. are newly reported species in this study. Suaeda glauca (Bunge) Bunge, Suaeda australis (R. Br.) Moq., Suaeda maritima (L.) Dumort., and Salicornia europaea L. are known as representative halophyte species of Chenopodiaceae. They were found in tidal flats, salt fields, and around the marina in Garyong-ri and Sinjang-ri.

\section{Endangered and rare plants}

The following nine taxa are classified as Threatened (critically endangered [CR], endangered [EN], vulnerable [VU]) and near threatened (NT) category in the Red Data Book of Endangered Vascular Plants in Korea (National Institute of Biological Resources, 2012) and the Rare Plants Data Book in Korea (Korea National Arboretum, 2008): Albizia calcareous (Roxb.) Prain, Wisteria japonica Siebold \& Zucc., Salomonia oblongifolia DC., Bupleurum falcatum L., Centranthera cochinchinensis (Lour.) Merr. var. lutea (H. Hara) H. Hara, Utricularia bifida L., Hololeion maximowiczii Kitam.,
Table 3. List of halophytes in Aphaedo Island.

\begin{tabular}{|c|c|}
\hline Family & Taxa \\
\hline Dryopteridaceae & Cyrtomium falcatum (L. f.) C. Presl 도깨비고비 ${ }^{a}$ \\
\hline Pinaceae & Pinus thunbergii Parl. 곰솔 \\
\hline \multirow[t]{8}{*}{ Chenopodiaceae } & Atriplex gmelinii C. A. Mey. 가는갯능쟁이 \\
\hline & Chenopodium glaucum L. 취명아주 \\
\hline & Salicornia europaea L. 퉁퉁마디 \\
\hline & Salsola komarovii Iljin 수송나물a \\
\hline & Suaeda australis (R. Br.) Moq. 방석나물 \\
\hline & Suaeda glauca (Bunge) Bunge 나문재 \\
\hline & Suaeda japonica Makino 칠면초 ${ }^{a}$ \\
\hline & Suaeda maritima (L.) Dumort. 해홍나물 \\
\hline Caryophyllaceae & Spergularia marina (L.) Griseb. 갯개미자리 \\
\hline Polygonaceae & Polygonum fusco-ochreatum Kom. 큰옥매듭풀a \\
\hline Primulaceae & Lysimachia mauritiana Lam. 갯까치수염 \\
\hline \multirow[t]{2}{*}{ Rosaceae } & Rosa rugosa Thunb. 해당화 \\
\hline & Rosa wichuraiana Crép. ex Déségl. 돌가시나무 \\
\hline Fabaceae & Lathyrus japonicus Willd. 갯완두a \\
\hline \multirow[t]{2}{*}{ Apiaceae } & Cnidium japonicum Miq. 갯사상자 \\
\hline & Glehnia littoralis F. Schmidt ex Miq. 갯방풍a \\
\hline Convolvulaceae & Calystegia soldanella (L.) Roem. \& Schult. 갯메꽃 \\
\hline Verbenaceae & Vitex rotundifolia L. f. 순비기나무 \\
\hline Labiatae & Scutellaria strigillosa Hemsl. 참골무꽃 ${ }^{2}$ \\
\hline \multirow[t]{3}{*}{ Asteraceae } & Artemisia capillaris Thunb. 사철쑥 \\
\hline & Aster hispidus Thunb. 갯쑥부쟁이 \\
\hline & Aster tripolium L. 갯개미취a \\
\hline Juncaginaceae & Triglochin maritimum L. 지채 \\
\hline Juncaceae & $\begin{array}{l}\text { Juncus setchuensis Buchenau var. effusoides } \\
\text { Buchenau 푸른갯골풀 }\end{array}$ \\
\hline \multirow[t]{6}{*}{ Cyperaceae } & $\begin{array}{l}\text { Bolboschoenus planiculmis (F. Schmidt) T. V. } \\
\text { Egorova 새섬매자기 }\end{array}$ \\
\hline & Carex kobomugi Ohwi 통보리사초 ${ }^{a}$ \\
\hline & Carex scabrifolia Steud. 천일사초 \\
\hline & $\begin{array}{l}\text { Eleocharis kamtschatica (C. A. Mey.) Kom. 올방개 } \\
\text { 아재비 }\end{array}$ \\
\hline & Fimbristylis longispica Steud. 큰하늘지기 \\
\hline & $\begin{array}{l}\text { Fimbristylis sieboldii Miq. Ex Franch. \& Sav. 갯하 } \\
\text { 늘지기 }\end{array}$ \\
\hline \multirow[t]{8}{*}{ Poaceae } & Calamagrostis epigeios (L.) Roth 산조풀a \\
\hline & Ischaemum anthephoroides (Steud.) Miq. 갯쇠보리 \\
\hline & Ischaemum crassipes (Steud.) Thell. 쇠보리 \\
\hline & Phragmites communis Trin. 갈대 \\
\hline & Polypogon monspeliensis (L.) Desf. 갯쇠돌피 \\
\hline & $\begin{array}{l}\text { Setaria viridis (L.) P. Beauv. var. pachystachys } \\
\text { (Franch. \& Sav.) Makino \& Nemoto 갯강아지풀 }\end{array}$ \\
\hline & Zoysia macrostachya Franch. \& Sav. 왕잔디 ${ }^{a}$ \\
\hline & Zoysia sinica Hance 갯잔디 \\
\hline Typhaceae & Typha laxmannii Lepech. 꼬마부들a \\
\hline
\end{tabular}

${ }^{\mathrm{a}}$ Newly identified taxa in this study. 
Table 4. List of endangered or rare plants in Aphaedo Island.

\begin{tabular}{|c|c|c|c|}
\hline \multirow{2}{*}{ Family } & \multirow{2}{*}{ Taxa } & \multicolumn{2}{|c|}{ Criteria $^{\mathrm{a}}$} \\
\hline & & $\mathrm{NIBR}^{\mathrm{b}}$ & $\mathrm{KNA}^{\mathrm{c}}$ \\
\hline \multirow[t]{2}{*}{ Fabaceae } & Albizia kalkora (Roxb.) Prain 왕자귀나무 & VU & EN \\
\hline & Wisteria japonica Siebold \& Zucc. 애기등 & - & VU \\
\hline Polygalaceae & Salomonia oblongifolia DC. 병아리다리 & VU & $\mathrm{CR}$ \\
\hline Apiaceae & Bupleurum falcatum L. 시호 & - & VU \\
\hline Scrophulariaceae & Centranthera cochinchinensis (Lour.) Merr. var. lutea (H. Hara) H. Hara 성주풀 & EN & VU \\
\hline Lentibulariaceae & Utricularia bifida L. 땅귀개 & - & VU \\
\hline Asteraceae & Hololeion maximowiczii Kitam. 께묵 & NT & EN \\
\hline \multirow[t]{2}{*}{ Orchidaceae } & Bletilla striata (Thunb.) Rchb. f. 자란 & - & VU \\
\hline & Pogonia japonica Rchb. f. 큰방울새란 & NT & VU \\
\hline
\end{tabular}

${ }^{\mathrm{a}} \mathrm{CR}$, critically endangered; EN, endangered; VU, vulnerable; NT, near threatened. ${ }^{b}$ Red data book of endangered vascular plants in Korea (National Institute of Biological Resources, 2012). ${ }^{\circ}$ Rare plants data book in Korea (Korean National Arboretum, 2008).
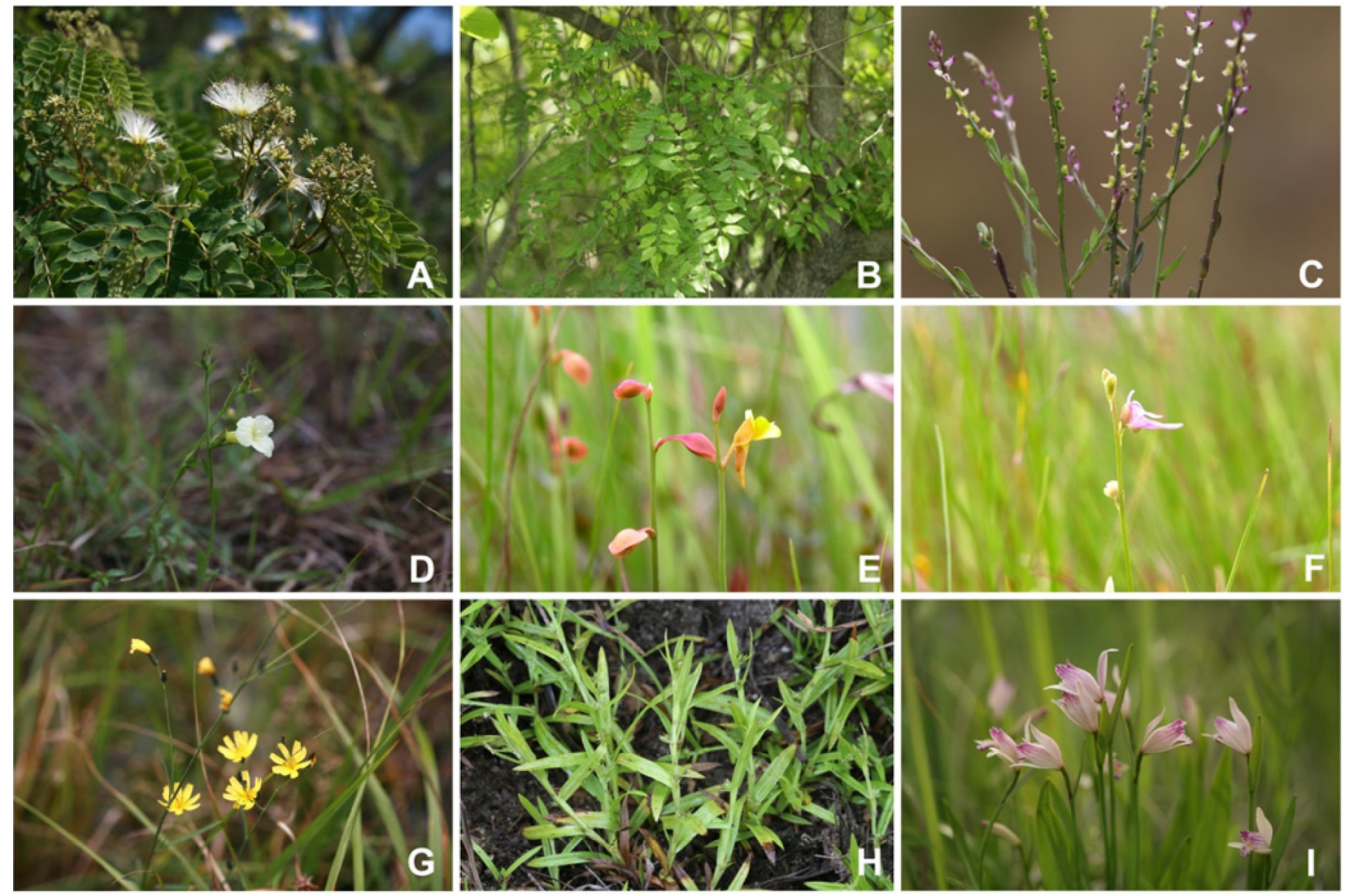

Fig. 2. Some remarkable plants in Aphaedo Island. A. Albizia kalkora (Roxb.) Prain. B. Wisteria japonica Siebold \& Zucc. C. Salomonia oblongifolia DC. D. Centranthera cochinchinensis (Lour.) Merr. var. lutea (H. Hara) H. Hara. E. Utricularia bifida L. F. Utricularia racemosa Wall. ex Walp. G. Hololeion maximowiczii Kitam. H. Scleria caricina (R. Br.) Benth. I. Pogonia japonica Rchb. f.

Bletilla striata (Thunb.) Rchb. f., and Pogonia japonica Rchb. f. (Table 4). Juniperus chinensis L. belongs to VU in the Red List category. However, it is excluded from the list as it is a planted species.
Albizia kalkora (Fig. 2A) was growing in foothills of Garyong-ri coast and in hills near Bunmae-ri ranch as reported in previous studies (Park, 2004; Jeong et al., 2011; Nam et al., 2012; Hwang et al., 2013). In addition, it has been found in 
uninhabited islands such as Songgongsan Mountain, Godongseom Island, Dolseom Island, Odo Island, Jeongjudo Island, and Haeduseom Island which belongs to Aphaedo Island (Hwang, 2017). Wisteria japonica (Fig. 2B) was observed in Hakgyo-ri Holmaesan Mountain area. Salomonia oblongifolia (Fig. 2C) was growing on the eastern slope of Bokryong-ri opposite to Holmaesan Mountain. Bupleurum falcatum was growing together in the native area of Albizia kalkora in Bunmae-ri. Centranthera cochinchinensis (Fig. 2D), Utricularia bifida (Fig. 2E), Hololeon maximowiczii (Fig. 2G), and Pogonia japonica (Fig. 2I) were distributed on the southern slope of Songgongsan Mountain bordering Songgong-ri and Daecheon-ri. In this slope, regional indicator species and a variety of mountain wetland plants have been identified, including V grade species Utricularia racemosa Wall. ex Walp. (Fig. 2F) and IV grade species such as Scleria caricina (R. Br.) Benth. (Fig. 2H), Scleria rugosa R. Br., Rhynchospora chinensis Nees \& Meyen, Scleria parvula Steud., Parnassia palustris L. var. multiseta Ledeb., Habenaria linearifolia Maxim., and so on. However, they were close to low-lying trails, causing concern about degradation of native habitat and disturbance of the ecosystem due to influx of invasive plants. There are also private forests and forests owned by families of same clan. Therefore, the possibility of habitat damage due to anthropogenic activity such as mountain clearing and installation of facilities cannot be excluded. In 2007, a bonsai park was constructed on the southern side of Songgongsan Mounatain, damaging much of the wetlands. However, in the upper area of the park, a wetland ecological garden was created and a part of it was preserved. In the future, it is necessary to conduct a detailed survey on wetlands distributed throughout Songgongsan Mountain and prepare conservation plans including designation of wetland protection areas.

\section{Regional indicator plants}

A total of 59 species and infraspecific taxa were confirmed as regional indicator species distributed in Aphaedo Island, including four grade V taxa [Albizia calcora (Roxb.) Prain, Wisteria japonica Siebold \& Zucc., Utricularia bifida L., Utricularia racemosa Wall. ex Walp.], six taxa in grade IV [including Polygonum fusco-ochreatum Kom., Salomonia oblongifolia DC., Centranthera cochinchinensis (Lour.) Merr. var. lutea (H. Hara), and H. Hara etc], 19 taxa in grade III [including Trichosanthes kirilowii Maxim. var. japonica (Miq.) Kitam., Cynanchum nipponicum Matsum., Bletilla striata (Thunb.) Rchb. f. etc.], four taxa in grade II, and 26 taxa in grade I. These taxa represent $13.1 \%$ of vascular plants identified on Aphaedo Island. A total of 24 taxa, including Polygonum fusco- ochreatum, Trichosanthes kirilowii, and Cynanchum nipponicum, are newly recorded regional indicator species (Table 5).

The majority of these regional indicator species in grade $\mathrm{V}$ and IV are endangered and rare plants of the Red Data Book of Endangered Vascular Plants in Korea (Table 4). Albizia calcora (Fig. 2A) is restricted to the western coast while Wisteria japonica (Fig. 2B) distributes relatively broad in the south coast and coastal areas in Jeollabuk-do. Both species are not currently protected by law. They are exposed to development threats because they are growing in the vicinity of villages (National Institute of Biological Resources, 2012). Polygonum fuscoochreatum, a halophyte reported in the western coast of south Chungcheong-do, was observed around the native habitat of Albizia kalkora on the coast of Garyong-ri. Cladium chinense Nees and Scleria caricina (R. Br.) Benth. (Fig. 2H) are restricted to the southern coastal regions and islands in Korea, respectively. In this study, they were found in the south coast of Songgongsan Mountain and wetlands of south slopes, respectively.

\section{Endemic plants}

The following endemic plants in the Korean Peninsula were identified in the survey area: Hepatica insularis Nakai, Indigofera koreana Ohwi, Lespedeza maritima Nakai, Forsythia koreana (Rdhder) Nakai, Paulownia coreana Uyeki, and Weigela subsessilis (Nakai) L. H. Bailey. This is equivalent to $1.6 \%$ of 360 species (including infraspecific taxa) of Korean endemic plants (Chung et al., 2017) (Table 6). Hepatica insularis was discovered in southern slope of Songgongsan Mountain while Indigofera koreana Ohwi was growing in the forests of Bunmae-ri and near Geumsansa Temple in Garyongri. Lespedeza maritima was distributed at the mountain foot of the north side of the Bokryong-ri Horae reservoir while Forsythia koreana (planted) was collected in the forests and farms near Horae reservoir. Paulownia coreana and Weigela subsessilis not previously reported on Aphaedo Island flora were observed in Bunmae-ri Moknaru and Songgongsan Mounatain north slopes, respectively.

\section{Naturalized plants and invasive species}

A total of 52 species and infraspecific taxa (in 16 families 40 genera) examined in this study are naturalized plants. This is equivalent to $11.5 \%$ of total vascular plants in Aphaedo Island. Among these, invasive (i.e., ecosystem disturbing) plant species include Rumex acetosella L., Aster pilosus Willd., Hypochaeris radicata L., Solidago altissima L., Paspalum distichum L., and P. distichum L. var. indutum Shinners (Table 7). A total of 334 taxa of naturalized plants listed in the 1st alien species management program of the Ministry of 
Table 5. List of regional indicator plants in Aphaedo Island.

\begin{tabular}{|c|c|c|}
\hline Grade & Taxa & No. of taxa \\
\hline V & $\begin{array}{l}\text { Albizia kalkora (Roxb.) Prain 왕자귀나무 } \\
\text { Wisteria japonica Siebold \& Zucc. 애기등 } \\
\text { Utricularia bifida } \text { L. 땅귀개 } \\
\text { Utricularia racemosa Wall. ex Walp. 이삭귀개 }\end{array}$ & 4 \\
\hline IV & $\begin{array}{l}\text { Platycladus orientalis (L.) Franco 측백나무(식재) } \\
\text { Polygonum fusco-ochreatum Kom. 큰옥매듭풀a } \\
\text { Salomonia oblongifolia DC. 병아리다리 } \\
\text { Centranthera cochinchinensis (Lour.) Merr. var. lutea (H. Hara) H. Hara 성주풀 } \\
\text { Cladium chinense Nees 층층고랭이 } \\
\text { Scleria caricina (R. Br.) Benth. 애기개올미 }\end{array}$ & 6 \\
\hline III & 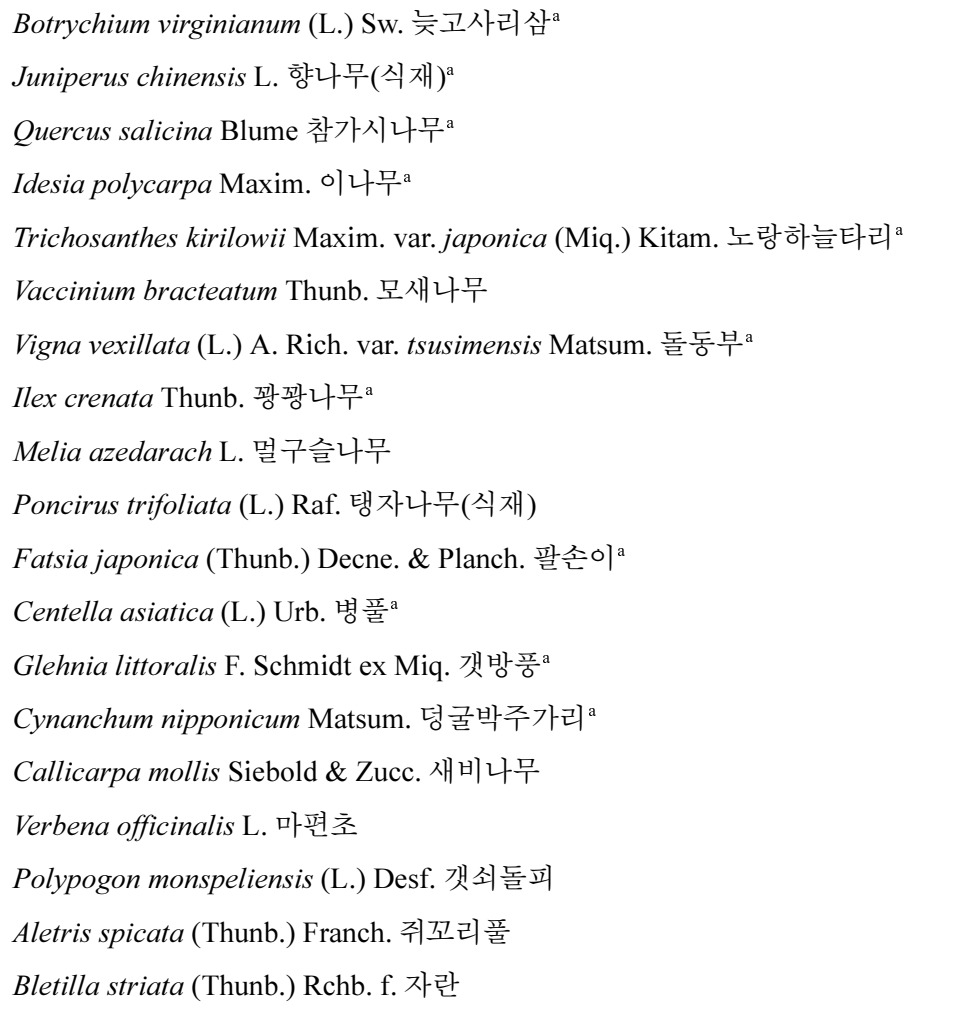 & 19 \\
\hline II & $\begin{array}{l}\text { Caltha palustris L. 동의나물 } \\
\text { Caryopteris incana (Thunb. ex Houtt.) Miq. 층꽃나무 } \\
\text { Ottelia alismoides (L.) Pers. 물질경이 } \\
\text { Epipactis thunbergii A. Gray 닭의난초 }\end{array}$ & 4 \\
\hline I & $\begin{array}{l}\text { Dicranopteris linearis (Burm. f.) Underw. 발풀고사리 } \\
\text { Lygodium japonicum (Thunb.) Sw. 실고사리 } \\
\text { Pteris multifida Poir. 봉의꼬리 }{ }^{\mathrm{a}} \\
\text { Thelypteris glanduligera (Kunze) Ching 사다리고사리 }{ }^{\mathrm{a}} \\
\text { Dryopteris erythrosora (D. C. Eaton) Kuntze 홍지네고사리 } \\
\text { Chloranthus fortunei (A. Gray) Sloms 옥녀꽃대 } \\
\text { Semiaquilegia adoxoides (DC.) Makino 개구리발톱 }\end{array}$ & 26 \\
\hline
\end{tabular}


Table 5. Continued.

\begin{tabular}{|c|c|c|}
\hline Grade & Taxa & No. of taxa \\
\hline \multirow[t]{19}{*}{ I } & Camellia japonica L. 동백나무 & 26 \\
\hline & Eurya japonica Thunb. 사스레피나무 & \\
\hline & Grewia parviflora Bunge 장구밥나무a & \\
\hline & Vaccinium oldhamii Miq. 정금나무 & \\
\hline & Lysimachia mauritiana Lam. 갯까치수염 & \\
\hline & Rubus hirsutus Thunb. 장딸기 ${ }^{a}$ & \\
\hline & Euonymus japonicus Thunb. 사철나무 & \\
\hline & Mallotus japonicus (L. f.) Müll. Arg. 예덕나무 & \\
\hline & Euscaphis japonica (Thunb.) Kanitz 말오줌때 & \\
\hline & Hedera rhombea (Miq.) Bean 송악a & \\
\hline & Calystegia soldanella (L.) Roem. \& Schult. 갯메꽃 ${ }^{a}$ & \\
\hline & Lithospermum zollingeri A. DC. 반디지치 & \\
\hline & Vitex rotundifolia L. f. 순비기나무 & \\
\hline & Scutellaria strigillosa Hemsl. 참골무꽃a & \\
\hline & Ligustrum japonicum Thunb. 광나무a & \\
\hline & Potamogeton cristatus Regel \& Maack 가는가래 ${ }^{a}$ & \\
\hline & Carex kobomugi Ohwi 통보리사초 ${ }^{a}$ & \\
\hline & Ischaemum anthephoroides (Steud.) Miq. 갯쇠보리a & \\
\hline & Sorghum nitidum (Vahl) Pers. 수수새 & \\
\hline
\end{tabular}

${ }^{a}$ Newly identified taxa in this study.

Table 6. List of plants endemic to Korea found in Aphaedo Island.

\begin{tabular}{ll}
\hline \hline \multicolumn{1}{c}{ Family } & Taxa \\
\hline Ranunculaceae & Hepatica insularis Nakai 새끼노루귀 \\
Fabaceae & $\begin{array}{l}\text { Indigofera koreana Ohwi 좀땅비싸리 } \\
\text { Lespedeza maritima Nakai 해변싸리 }\end{array}$ \\
Oleaceae & Forsythia koreana (Rdhder) Nakai 개나리(식재) \\
Scrophulariaceae & Paulownia coreana Uyeki 오동나무 \\
Diervillaceae & Weigela subsessilis (Nakai) L. H. Bailey 병꽃나무 \\
\hline
\end{tabular}

Environment (Bang, 2014) are divided into four categories based on risk level: ecosystem risk, ecosystem risk concern, harmless to ecosystem, and unclassified. According to the risk level, naturalized plants of Aphaedo Island are classified as follows. Ecosystem risk species includs all six invasive species listed above. Ecosystem risk concern species were Phytolacca americana L., Plantago lanceolata L., and Aster subulatus Michx.. Thirteen species were included in harmless to ecosystem category while 29 species were classified in the unclassified category. On the other hand, Indigofera bungeana Walp. not inlcuded in the list of the Ministry of Environment is a plant introduced from China for landslide prevention as well as greening purpose. It was included in the list of naturalized plants of this study.

Naturalized plants (species and infraspecific taxa) surveyed in Aphaedo Island since 2003 include 11 taxa by Park (2004), 67 taxa (a total of 68 taxa are presented, of which Veronica polita Fr. subsp. lilacina (H. Hara ex T. Yamaz.) T. Yamaz. are excluded here because they are native plants) by Kim (2008), 16 taxa by Hwang et al. (2013), 10 taxa by Jeong et al. (2011), and 55 taxa by Nam et al. (2012). The total number of naturalized plants reported in Aphaedo Island reached a total 
of 22 families, 77 genera, 100 taxa (species and infraspecific taxa), including 12 newly confirmed ones in this study such as Malva neglecta Wallr., Aster pilosus, and Briza minor L. etc. (Tables 7,8). This corresponds to $14.7 \%$ of 680 taxa (excluding cultivated plants) of vascular plants distributed on Aphaedo Island to date. The percentage of naturalized plants (naturalization rate) in Aphaedo Island is higher than that of
Imjado Island (5.5\%) (Hong et al., 2011), Dochodo Island (8.9\%) (Nam et al., 2012), and Bigeumdo Island (9.6\%) (Nam et al., 2012). In addition, this is higher than the average naturalization rate $(7.66 \%)$ of plants in inhabited island of Jeollanam-do and the naturalization rate of plants on islands in the Yellow Sea (8.39\%) (Kim et al., 2017). The naturalization rate of plants on inhabited islands has a

Table 7. List of naturalized plants in Aphaedo Island.

\begin{tabular}{|c|c|c|c|c|c|c|c|c|}
\hline \multirow{2}{*}{ Family } & \multirow{2}{*}{ Taxa } & \multirow{2}{*}{$\mathrm{D}^{\mathrm{a}}$} & \multicolumn{6}{|c|}{ Study $^{\mathrm{b}}$} \\
\hline & & & 1 & 2 & 3 & 4 & 5 & 6 \\
\hline Saururaceae & Houttuynia cordata Thunb. 약모밀 & 4 & & & & $\mathrm{O}$ & & \\
\hline Phytolaccaceae & Phytolacca americana L. 미국자리공 & 2 & & $\mathrm{O}$ & $\mathrm{O}$ & $\mathrm{O}$ & $\mathrm{O}$ & $\mathrm{O}$ \\
\hline \multirow[t]{4}{*}{ Chenopodiaceae } & Atriplex hastata L. 창명아주 & 4 & & & & $\mathrm{O}$ & & \\
\hline & Chenopodium album L. 흰명아주 & 4 & & $\mathrm{O}$ & & $\mathrm{O}$ & & \\
\hline & Chenopodium ficifolium $\mathrm{Sm}$. 좀명아주 & 4 & & & $\mathrm{O}$ & $\mathrm{O}$ & $\mathrm{O}$ & $\mathrm{O}$ \\
\hline & Chenopodium glaucum L. 취명아주 & 4 & & $\mathrm{O}$ & & & & $\mathrm{O}$ \\
\hline \multirow[t]{2}{*}{ Amaranthaceae } & Amaranthus lividus L. 개비름 & 3 & & & & & & $\mathrm{O}$ \\
\hline & Amaranthus viridis L. 청비름 & 3 & & $\mathrm{O}$ & & $\mathrm{O}$ & & \\
\hline \multirow[t]{3}{*}{ Caryophyllaceae } & Cerastium glomeratum Thuill. 양점나도나물 & 4 & & $\mathrm{O}$ & & $\mathrm{O}$ & & $\mathrm{O}$ \\
\hline & Spergula arvensis L. 들개미자리 & 3 & & & $\mathrm{O}$ & & $\mathrm{O}$ & $\mathrm{O}$ \\
\hline & Spergularia rubra J. Presl \& C. Presl 유럽개미자리 & 3 & & $\mathrm{O}$ & & $\mathrm{O}$ & & \\
\hline \multirow[t]{5}{*}{ Polygonaceae } & Fallopia convolvulus (L.) Á. Löve 나도닭의덩굴 & 4 & & $\mathrm{O}$ & & & & \\
\hline & Rumex acetosella L. 애기수영 ${ }^{\circ}$ & 1 & & $\mathrm{O}$ & $\mathrm{O}$ & & $\mathrm{O}$ & $\mathrm{O}$ \\
\hline & Rumex crispus L. 소리쟁이 & 4 & $\mathrm{O}$ & $\mathrm{O}$ & $\mathrm{O}$ & $\mathrm{O}$ & $\mathrm{O}$ & $\mathrm{O}$ \\
\hline & Rumex nipponicus Franch. \& Sav. 좀소리쟁이 & 4 & & & & & & $\mathrm{O}$ \\
\hline & Rumex obtusifolius L. 돌소리쟁이 & 2 & & $\mathrm{O}$ & & $\mathrm{O}$ & & \\
\hline \multirow[t]{3}{*}{ Malvaceae } & Abutilon theophrasti Medik. 어저귀 & 3 & & $\mathrm{O}$ & & & & \\
\hline & Malva neglecta Wallr. 난쟁이아욱 & 4 & & & & & & $\mathrm{O}$ \\
\hline & Malva sylvestris L. var. mauritiana (L.) Boiss. 당아욱 & 3 & & $\mathrm{O}$ & & & & \\
\hline \multirow[t]{4}{*}{ Brassicaceae } & Brassica juncea (L.) Czern. 갓 & 4 & & $\mathrm{O}$ & & $\mathrm{O}$ & $\mathrm{O}$ & $\mathrm{O}$ \\
\hline & Lepidium apetalum Willd. 다닥냉이 & 4 & & $\mathrm{O}$ & & & & $\mathrm{O}$ \\
\hline & Lepidium virginicum L. 콩다닥냉이 & 4 & & $\mathrm{O}$ & $\mathrm{O}$ & $\mathrm{O}$ & $\mathrm{O}$ & $\mathrm{O}$ \\
\hline & Thlaspi arvense L. 말냉이 & 4 & & $\mathrm{O}$ & & & & \\
\hline Rosaceae & Potentilla supina L. 개소시랑개비 & 3 & & $\mathrm{O}$ & & & & \\
\hline \multirow[t]{8}{*}{ Fabaceae } & Amorpha fruticosa L. 족제비싸리 & 2 & & $\mathrm{O}$ & & & & \\
\hline & Indigofera bungeana Walp. 큰낭아초 & - & & & & & & $\mathrm{O}$ \\
\hline & Medicago lupulina L. 잔개자리 & 4 & & $\mathrm{O}$ & & $\mathrm{O}$ & & \\
\hline & Medicago polymorpha L. 개자리 & 4 & & & & $\mathrm{O}$ & & $\mathrm{O}$ \\
\hline & Melilotus suaveolens Ledeb. 전동싸리 & 3 & & $\mathrm{O}$ & & & & \\
\hline & Robinia pseudoacacia L. 아까시나무 & 4 & $\mathrm{O}$ & $\mathrm{O}$ & & $\mathrm{O}$ & & $\mathrm{O}$ \\
\hline & Trifolium repens $\mathrm{L}$. 토끼풀 & 4 & $\mathrm{O}$ & $\mathrm{O}$ & & $\mathrm{O}$ & & $\mathrm{O}$ \\
\hline & Vicia villosa Roth 벳지 & 3 & & & & $\mathrm{O}$ & & $\mathrm{O}$ \\
\hline
\end{tabular}


Table 7. Continued.

\begin{tabular}{|c|c|c|c|c|c|c|c|c|}
\hline \multirow{2}{*}{ Family } & \multirow{2}{*}{ Taxa } & \multirow{2}{*}{$\mathrm{D}^{\mathrm{a}}$} & \multicolumn{6}{|c|}{ Study $^{b}$} \\
\hline & & & 1 & 2 & 3 & 4 & 5 & 6 \\
\hline \multirow[t]{2}{*}{ Onagraceae } & Oenothera biennis L. 달맞이꽃 & 3 & $\mathrm{O}$ & $\mathrm{O}$ & & $\mathrm{O}$ & & $\mathrm{O}$ \\
\hline & Oenothera glazioviana Micheli 큰달맞이꽃 & 3 & & & & & & $\mathrm{O}$ \\
\hline Euphorbiaceae & Euphorbia supina Raf. 애기땅빈대 & 4 & & $\mathrm{O}$ & & $\mathrm{O}$ & $\mathrm{O}$ & $\mathrm{O}$ \\
\hline Oxalidaceae & Oxalis articulata Sabigny 덩이괭이밥 & 3 & & $\mathrm{O}$ & & & & \\
\hline \multirow[t]{2}{*}{ Solanaceae } & Datura tatula L. 독말풀 & 4 & & $\mathrm{O}$ & & & & \\
\hline & Physalis angulata L. 땅꽈리 & 4 & & & & $\mathrm{O}$ & & \\
\hline \multirow[t]{5}{*}{ Convolvulaceae } & Cuscuta pentagona Engelm. 미국실새삼 & 4 & & & & & & $\mathrm{O}$ \\
\hline & Ipomoea hederacea Jacq. 미국나팔꽃 & 4 & & $\mathrm{O}$ & & $\mathrm{O}$ & & \\
\hline & Ipomoea hederacea Jacq. var. integriuscula A. Gray 둥근잎미국나팔꽃 & 4 & & & & $\mathrm{O}$ & & \\
\hline & Ipomoea purpurea (L.) Roth 둥근잎나팔꽃 & 4 & & & & $\mathrm{O}$ & & $\mathrm{O}$ \\
\hline & Quamoclit coccinea Moench 둥근잎유홍초 & 3 & & & & & $\mathrm{O}$ & \\
\hline Boraginaceae & Symphytum officinale L. 컴프리 & 4 & & & & $\mathrm{O}$ & & \\
\hline Plantaginaceae & Plantago lanceolata L. 창질경이 & 2 & $\mathrm{O}$ & $\mathrm{O}$ & & $\mathrm{O}$ & & $\mathrm{O}$ \\
\hline \multirow[t]{2}{*}{ Scrophulariaceae } & Veronica arvensis L. 선개불알풀 & 4 & & & & $\mathrm{O}$ & & $\mathrm{O}$ \\
\hline & Veronica persica Poir. 큰개불알풀 & 4 & & $\mathrm{O}$ & & $\mathrm{O}$ & $\mathrm{O}$ & $\mathrm{O}$ \\
\hline \multirow[t]{24}{*}{ Asteraceae } & Ambrosia artemisiifolia $\mathrm{L}$. 돼지풀c & 1 & $\mathrm{O}$ & $\mathrm{O}$ & & $\mathrm{O}$ & & \\
\hline & Aster pilosus Willd. 미국쑥부쟁이들 & 1 & & & & & & $\mathrm{O}$ \\
\hline & Aster subulatus Michx. 비짜루국화 & 2 & & & & $\mathrm{O}$ & & $\mathrm{O}$ \\
\hline & Aster subulatus Michx. var. sandwicensis A. G. Jones 큰비짜루국화 & 2 & & $\mathrm{O}$ & & $\mathrm{O}$ & & \\
\hline & Bidens frondosa L. 미국가막사리 & 2 & & & & $\mathrm{O}$ & $\mathrm{O}$ & \\
\hline & Bidens pilosa L. 울산도깨비바늘 & 4 & & & & & & $\mathrm{O}$ \\
\hline & Carduus crispus L. 지느러미엉겅퀴 & 4 & & & & $\mathrm{O}$ & & \\
\hline & Chrysanthemum leucanthemum L. 불란서국화 & 4 & & & & $\mathrm{O}$ & & \\
\hline & Conyza bonariensis (L.) Cronquist 실망초 & 4 & & $\mathrm{O}$ & & & & $\mathrm{O}$ \\
\hline & Conyza canadensis (L.) Cronquist 망초 & 4 & & $\mathrm{O}$ & & & & $\mathrm{O}$ \\
\hline & Conyza sumatrensis (Retz.) E. Walker 큰망초 & 4 & & $\mathrm{O}$ & & $\mathrm{O}$ & & \\
\hline & Coreopsis lanceolata L. 큰금계국 & 3 & $\mathrm{O}$ & $\mathrm{O}$ & & $\mathrm{O}$ & & $\mathrm{O}$ \\
\hline & Coreopsis tinctoria Nutt. 기생초 & 3 & & $\mathrm{O}$ & & & & \\
\hline & Cosmos bipinnatus Cav. 코스모스 & 3 & $\mathrm{O}$ & $\mathrm{O}$ & & & & \\
\hline & Cosmos sulphureus Cav. 노랑코스모스 & 3 & & $\mathrm{O}$ & & & & \\
\hline & Crassocephalum crepidioides (Benth.) S. Moore 주홍서나물 & 3 & $\mathrm{O}$ & & & & & $\mathrm{O}$ \\
\hline & Erechtites hieraciifolia (L.) Raf. ex DC. 붉은서나물 & 3 & & $\mathrm{O}$ & & & & $\mathrm{O}$ \\
\hline & Erigeron annuus (L.) Pers. 개망초 & 4 & $\mathrm{O}$ & $\mathrm{O}$ & $\mathrm{O}$ & $\mathrm{O}$ & $\mathrm{O}$ & $\mathrm{O}$ \\
\hline & Galinsoga parviflora Cav. 별꽃아재비 & 4 & & $\mathrm{O}$ & & & & \\
\hline & Gamochaeta calviceps (Fernald) Cabrera 선풀솜나물 & 4 & & & & $\mathrm{O}$ & & \\
\hline & Helianthus tuberosus L. 뚱딴지 & 3 & & $\mathrm{O}$ & & & & \\
\hline & 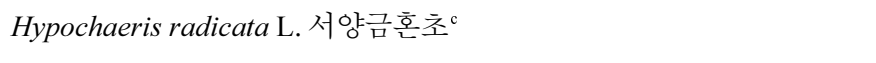 & 1 & & & & $\mathrm{O}$ & & $\mathrm{O}$ \\
\hline & Lactuca scariola L. 가시상추c & 1 & & $\mathrm{O}$ & & $\mathrm{O}$ & & \\
\hline & Rudbeckia hirta L. var. pulcherrima Farw. 원추천인국 & 4 & & $\mathrm{O}$ & & & & \\
\hline
\end{tabular}


Table 7. Continued.

\begin{tabular}{|c|c|c|c|c|c|c|c|c|}
\hline \multirow{2}{*}{ Family } & \multirow{2}{*}{ Taxa } & \multirow{2}{*}{$\mathrm{D}^{\mathrm{a}}$} & \multicolumn{6}{|c|}{ Study $^{\mathrm{b}}$} \\
\hline & & & 1 & 2 & 3 & 4 & 5 & 6 \\
\hline & Senecio vulgaris L. 개쑥갓 & 4 & & $\mathrm{O}$ & $\mathrm{O}$ & $\mathrm{O}$ & $\mathrm{O}$ & $\mathrm{O}$ \\
\hline & Solidago altissima L. 양미역취` & 1 & & $\mathrm{O}$ & & & & $\mathrm{O}$ \\
\hline & Solidago gigantea Aiton subsp. serotina (Aiton) McNeill 미국미역취 & 4 & & & & & & $\mathrm{O}$ \\
\hline & Sonchus asper (L.) Hill 큰방가지똥 & 3 & & $\mathrm{O}$ & & $\mathrm{O}$ & & $\mathrm{O}$ \\
\hline & Sonchus oleraceus L. 방가지똥 & 3 & & $\mathrm{O}$ & $\mathrm{O}$ & $\mathrm{O}$ & $\mathrm{O}$ & $\mathrm{O}$ \\
\hline & Tagetes minuta L. 만수국아재비 & 2 & & $\mathrm{O}$ & & & & \\
\hline & Taraxacum laevigatum (Willd.) DC. 붉은씨서양민들레 & 4 & & $\mathrm{O}$ & & & & \\
\hline & Taraxacum officinale F. H. Wigg. 서양민들레 & 4 & & $\mathrm{O}$ & & & & $\mathrm{O}$ \\
\hline & Xanthium canadense Mill. 큰도꼬마리 & 4 & & $\mathrm{O}$ & & & & \\
\hline & Xanthium strumarium L. 도꼬마리 & 4 & & $\mathrm{O}$ & & & & \\
\hline Commelinaceae & Tradescantia ohiensis Raf. 자주달개비 & 3 & & $\mathrm{O}$ & & & & \\
\hline \multirow[t]{16}{*}{ Poaceae } & Alopecurus japonicus Steud. 털뚝새풀 & 4 & & & & & & $\mathrm{O}$ \\
\hline & Avena fatua L. 메귀리 & 4 & & $\mathrm{O}$ & & $\mathrm{O}$ & & $\mathrm{O}$ \\
\hline & Briza minor L. 방울새풀 & 3 & & & & & & $\mathrm{O}$ \\
\hline & Bromus rigidus Roth 긴까락빕새귀리 & 4 & & & & & & $\mathrm{O}$ \\
\hline & Bromus unioloides Kunth 큰이삭풀 & 3 & & $\mathrm{O}$ & & $\mathrm{O}$ & & $\mathrm{O}$ \\
\hline & Dactylis glomerata L. 오리새 & 4 & & $\mathrm{O}$ & & $\mathrm{O}$ & & $\mathrm{O}$ \\
\hline & Eragrostis curvula (Schrad.) Nees 능수참새그령 & 4 & & $\mathrm{O}$ & & & & \\
\hline & Festuca arundinacea Schreb. 큰김의털 & 2 & & $\mathrm{O}$ & & $\mathrm{O}$ & & \\
\hline & Festuca myuros L. 들묵새 & 4 & & $\mathrm{O}$ & & $\mathrm{O}$ & & \\
\hline & Leptochloa malabarica (L.) Veldkamp 갯드렁새 & 4 & & $\mathrm{O}$ & & $\mathrm{O}$ & & \\
\hline & Lolium multiflorum Lam. 쥐보리 & 4 & & $\mathrm{O}$ & $\mathrm{O}$ & $\mathrm{O}$ & $\mathrm{O}$ & $\mathrm{O}$ \\
\hline & Lolium perenne L. 호밀풀 & 4 & & $\mathrm{O}$ & & $\mathrm{O}$ & & \\
\hline & Panicum dichotomiflorum Michx. 미국개기장 & 4 & & $\mathrm{O}$ & & $\mathrm{O}$ & & \\
\hline & Paspalum distichum L. 물참새피 ${ }^{c}$ & 1 & & $\mathrm{O}$ & & $\mathrm{O}$ & & $\mathrm{O}$ \\
\hline & Paspalum distichum L. var. indutum Shinners 털물참새피 ${ }^{\mathfrak{}}$ & 1 & & $\mathrm{O}$ & & $\mathrm{O}$ & & $\mathrm{O}$ \\
\hline & Poa pratensis L. 왕포아풀 & 4 & & $\mathrm{O}$ & & $\mathrm{O}$ & $\mathrm{O}$ & \\
\hline \multirow[t]{3}{*}{ Iridaceae } & Iris pseudoacorus L. 노랑꽃창포 & 4 & & & & $\mathrm{O}$ & & \\
\hline & Sisyrinchium angustifolium Mill. 등심붓꽃 & 3 & $\mathrm{O}$ & & & & & $\mathrm{O}$ \\
\hline & Total & & 11 & 67 & 10 & 55 & 16 & 52 \\
\hline
\end{tabular}

${ }^{\mathrm{a})} \mathrm{D}$, degree of ecological risk (Bang, 2014). ${ }^{\text {b) }}$, Park (2004); 2, Kim (2008); 3, Jeong et al. (2011); 4, Nam et al. (2012); 5, Hwang et al. (2013); 6, This study. ${ }^{\circ}$ Ecosystem disturbing plants (National Institute of Environmental Research, 2012).

significant positive correlation with the size of the island (Kim et al., 2017). The naturalization rate of plants on Aphaedo Island is high compared to the naturalization rate of plants on Daebudo Island (12.9\%) (Lim et al., 2014) which is adjacent to the metropolitan area and under accelerated development.

Aphaedaegyo is a bridge connecting Aphaedo Island and Mokpo City. It was started in 2000 and completed in 2008.
Naturalized plants of Aphaedo Island were increased by a factor of 1.4 compared to that at the beginning period of the bridge construction (11 taxa) and 9 times just before the onset (69 taxa) (Table 8). On the other hand, most of invasive plants identified in this survey were found in the vicinity of Sinjangri, the place where it was connected with the Aphaedaegyo. Aster pilosus, Hypochaeris radicata, and Solidago altissima 
Table 8. Change in number of naturalized plants in Aphaedo Island.

\begin{tabular}{lcccc}
\hline \hline \multirow{2}{*}{ Survey year } & \multicolumn{3}{c}{ Taxa } \\
\cline { 2 - 5 } & Total (ratio, \%) & Newly identified & Re-identified & Cumulative total \\
\hline 2003 (Park, 2004) & $11(7.1)$ & - & - & 11 \\
2006-2007 (Kim, 2008) & $67(+)$ & 58 & 9 & 69 \\
2007-2010 (Hwang et al., 2013) & $16(5.6)$ & 4 & 12 & 73 \\
2010 (Jeong et al., 2011) & $10(9.8)$ & - & 10 & 73 \\
2011 (Nam et al., 2012) & $55(14.4)$ & 15 & 40 & 88 \\
2011-2016 (this study) & $52(11.5)$ & 40 & 100 \\
\hline 2003-2016 (total) & $100(14.2)$ & &
\end{tabular}

+ , all naturalized plants surveyed.

${ }^{a}$ Naturalized plants ratio in each study.

were frequently observed near the marina and Route 2. Paspalum distichum and P. distichum var. indutum were found near the reservoir (Ohoje) located in the northwest of the Aphaedaegyo and in a nearby waterway. On the other hand, Rumex acetosella and Hypochaeris radicata were distributed sporadically along the southern slope of Songgongsan Mountain where rare plants such as Utricularia bifida, Centranthera cochinchinensis, and Pogonia japonica were found.

Once the Saecheonnyeondaegyo is open in 2018 and Amtaedo Island, Jaeundo Island, Palgeumdo Island, and Anjwado Island are connected to Aphaedo Island, it is expected that naturalized plants of Aphaedo Island will rapidly flow into these areas. Island areas are vulnerable to external influx and environmental changes due to limited habitat space (Kim et al., 2017). From this perspective, it is necessary to closely monitor the naturalized plants on these islands.

\section{Conflict of Interest}

Authors declare that there is no conflict of interest.

\section{Acknowledgments}

This work was supported by a grant (NIBR201701202) from the National Institute of Biological Resources (NIBR) funded by the Ministry of Environment (MOE), Republic of Korea.

\section{Literature Cited}

Bang, S. W. 2014. A Study for Mid to Long-term Management of Alien Species. Ministry of Environment, Sejong, 295 pp. (in Korean) Chung, G. Y., K. S. Chang, J.-M. Chung, H. J. Choi, W.-K. Paik and J.-O. Hyun. 2017. A checklist of endemic plants on the Korean
Peninsula. Korean Journal of Plant Taxonomy 47: 264-288. (in Korean)

Hong, H.-H., H.-D. Son, S. In and H.-T. Im. 2011. Floristic study of Inmja-do (Isl.). Korean Journal of Plant Taxonomy 41: 429439. (in Korean)

Hoshino, T. and T. Masaki. 2011. Illustrated Sedges of Japan. Heibonsha, Tokyo, $778 \mathrm{pp}$.

Hwang, H.-R. 2017. Distribution characteristics and status of the Albizia kalkora (Roxb.) Prain in Mokpo. MS thesis, Chonnam University, Gwangju, 64 pp. (in Korean)

Hwang, H.-S., J.-C. Yang, S.-H. Oh, Y.-M. Lee and K.-S. Chang. 2013. A study on the flora of 15 islands in the western sea of Jeollanamdo Province, Korea. Journal of Asia-Pacific Biodiversity 6: $281-310$.

Jeong, J. H., C. M. Jang, K. H. Kim, Y. J. Oh and W. K. Paik. 2011. Flora of Sinan-gun (Sinan, Jeollanam-do, Korea). Korean Journal of Nature Conservation 5: 107-134. (in Korean)

Kim, H.-H., D.-B. Kim, C.-H. Jeon, C.-S. Kim and W.-S. Kong. 2017. Island-biogeographical characteristics of naturalized plant in Jeollanamdo islands. Journal of Environmental Impact Assessment 26: 272-289. (in Korean)

Kim, H. S. 2008. Ecological studies on the changes of distribution of plants in coastal islands: a case of the Abhaedo, Shinangun. Journal of Korean Island 20: 79-88. (in Korean)

Korea National Arboretum. 2008. Rare Plants Data Book in Korea. Geobook Publishing Co., Pocheon, 332 pp. (in Korean).

Korea National Arboretum and The Plant Taxonomic Society of Korea. 2007. A Synonymic List of Vascular Plants in Korea. Korea National Arboretum, Pocheon, 534 pp. (in Korean)

Lee, B.-Y., G.-H. Nam, J.-H. Yun, G. Y. Cho, J. S. Lee, J.-H. Kim, T. S. Park, K. Kim and K. Oh. 2010. Biological indicators to monitor responses against climate change in Korea. Korean Journal of Plant Taxonomy 40: 202-207. 
Lee, T. B. 2003. Coloured Flora of Korea. Vol. I, II. Hyangmunsa, Seoul, Vol. 1, 914 pp, Vol. II, 910 pp. (in Korean)

Lee, W. T. 1996a. Lineamenta Florae Koreae. Academy Press, Seoul, 1688 pp. (in Korean)

Lee, W. T. 1996b. Standard Illustrations of Korean plants. Academy Press, Seoul, 624 pp. (in Korean)

Lee, W. T. and Y. J. Yim. 2002. Plant Geography with Special Reference to Korea. Kangwon National University Press, Chuncheon, 412 pp. (in Korean)

Lee, Y. N. 2006. New flora of Korea. Vol. I, II. Gyohaksa, Seoul, Vol. 1, 975 pp, Vol. 2, 885 pp. (in Korean)

Lim, Y., K.-P. Yoo, The Korean Society of Plant Parataxonomists and J.-O. Hyun. 2014. Floristic study of Daebudo Island. Korean Journal of Plant Resources 27: 447-476. (in Korean)

Na, H. R. and J.-O. Hyun. 2015. A checklist of halophyte species in Korea. In 2015 International Symposium on Plant Sciences \& Annual Conference of the Korean Society of Plant Biologists. Korean Society of Plant Biologists, Seoul. P. 217.

Nam, C.-H., S.-H. Park, S.-Y. Jung, S. H. Oh, J.-O. Hyun, H. J. Kwon and K. S. Chang. 2012. The vascular plants of Sinan-gun Jellanam-do Korea: Aphae-do (Is.), Bigeum-do (Is.) and Dochodo Island. Journal of Korea Nature 5: 65-87.

National Geographic Information Institute. 2010. The Origin of Korean Geographical Names: Jeonnam and Jeju. National Geographic Information Institute, Suwon. Pp. 695-721. (in Korean)

National Institute of Biological Resources. 2012. Red Data Book of Endangered Vascular Plants in Korea. National Institute of Biological Resources, Incheon, 167 pp. (in Korean)

National Institute of Biological Resources. 2017. National List of Species of Korea, 2016. Retrieved Mar. 1, 2018, available from http://www.kbr.go.kr.
National Institute of Environmental Research. 2012. A Guide to the 4th National Natural Environment Research. National Institute of Environmental Research, Incheon. Pp. 173-226. (in Korean).

Oh, B. U., D. G. Jo, B. Y. Sun, B. H. Choi, J. H. Pak, H. T. Im, C. S. Chang, W. K. Paik, G. Y. Chung, K. R. Park, J. H. Kim and C. G. Jang. 2004. Distribution Maps of Vascular Plants of Korean Peninsula, I. South-Coast Province. Korea National Arboretum, Pocheon, 692 pp. (in Korean)

Oh, B. U., D. G. Jo, S. C. Ko, B. H. Choi, W. K. Paik, G. Y. Chung, Y. M. Lee and C. G. Jang. 2010. 300 Target Plants Adaptable to Climate Change in the Korean Peninsula. Korea National Arboretum, Pocheon, 492 pp. (in Korean)

Park, C.-W. 2007. The Genera of Vascular Plants of Korea. Flora of Korea Editorial Committee. Academy Publishing Co., Seoul, 1482 pp.

Park, S. H. 2009. New Illustrations and Photographs of Naturalized Plants of Korea. Ilchokak, Seoul, 575 pp. (in Korean)

Park, S. H., Y. M. Lee, S. Y. Jeong, G. S. Jang, W. C. Kang, S. S. Jeong, S. H. Oh and J. C. Yang. 2011. Illustrated Grasses of Korea. Revised and enlarged edition. Korea National Arboretum, Pocheon, 600 pp. (in Korean)

Park, S. H., Y. M. Lee, J. C. Yang, D. K. Jo, G. H. Lee, C. S. Chang, H. J. Lee, H. J. Choi, S. S. Jeong and J. H. Lee. 2008. Illustrated Pteridophyta of Korea. Korea National Arboretum, Pocheon, 547 pp. (in Korean)

Park, Y. K. 2004. Flora of islands in west and south regions of Korea. MS thesis, Honam University, Gwangju, 121 pp. (in Korean)

Shinan-gun Office. 2016. Shinan Statistical Year Book. Vol. 56, II. Land and Climate. Shinan-gun Office, Shinan. Pp. 47-58. (in Korean)

\section{압해도(전라남도 신안군)의 식물상 \\ 현진오* - 나혜련 · 김연수 · 한병우 \\ 동북아생물다양성연구소}

적 요: 전라남도 신안군에 위치한 압해도의 관속식물상을 조사하고 분포가 확인된 주요 식물에 대해 논의 하였다. 2011년 5월부터 2016년 3월까지 총 28일간 현지조사를 통해 확보된 표본에 근거하여 식물목록을 작 성한 결과, 압해도에 분포하는 관속식물은 102과 294속에 속하는 413종 6아종 30변종 2품종 등 총 451분류 군으로 정리되었다. 이 중에서 멸종위기 및 희귀식물은 왕자귀나무, 병아리다리, 성주풀 등 9분류군이 확인 되었다. 식물구계학적 특정식물은 $\mathrm{V}$ 등급 4분류군, IV등급 6분류군, III등급 19분류군, II등급 4분류군, I등급 26 분류군 등 총 59 분류군이 조사되었다. 특산식물은 새끼노루귀, 좀땅비싸리, 해변싸리 등 6 분류군이 확인되 었다. 귀화식물은 생태계교란식물인 애기수영, 미국쑥부쟁이, 서양금혼초 등을 포함하여 총 52 분류군으로 나 타났다. 압해대교 개통 전후에 수행된 선행연구 비교를 통하여 압해도의 귀화식물 변화를 분석하였다.

주요어: 압해도, 식물상, 멸종위기 및 희귀식물, 식물구계학적 특정식물, 특산식물, 귀화식물 
Appendix 1. The list of vascular plants of Aphaedo Island.

\begin{tabular}{|c|c|c|c|c|c|c|c|}
\hline \multirow{2}{*}{ No. } & \multirow{2}{*}{$\begin{array}{c}\text { Taxa and representative voucher specimen number } \\
\text { (KP: KOSPVP0000-, KN: KONPVP0000-, NP: NIBRVP0000-) }\end{array}$} & \multicolumn{6}{|c|}{ Study } \\
\hline & & 1 & 2 & 3 & 4 & 5 & 6 \\
\hline
\end{tabular}

Ophioglossaceae 고사리삼과

1 Botrychium virginianum (L.) Sw. 늦고사리삼 KP256149

Osmundaceae 고비과

2 Osmunda japonica Thunb. 고비 KP255588

Gleicheniaceae 발풀고사리과

3 Dicranopteris linearis (Burm. f.) Underw. 발풀고사리 NP582539

$\begin{array}{lll}\mathrm{O} & \mathrm{O} & \mathrm{O}\end{array}$

Schizaeaceae 실고사리과

4 Lygodium japonicum (Thunb.) Sw. 실고사리 KP255667

O $\quad \mathrm{O} \quad \mathrm{O}$

Pteridaceae 봉의꼬리과

5 Pteris multifida Poir. 봉의꼬리 KP256151

Dennstaedtiaceae 잔고사리과

6 Pteridium aquilinum (L.) Kuhn var. latiusculum (Desv.) Underw. ex A. Heller 고사리 KN373336 Aspleniaceae 꼬리고사리과

7 Asplenium incisum Thunb. 꼬리고사리 KP256153

Athyriaceae 개고사리과

8 Deparia japonica (Thunb.) M. Kato 진고사리 KP256155

Thelypteridaceae 처녀고사리과

9 Thelypteris glanduligera (Kunze) Ching 사다리고사리 KP256161

10 Thelypteris palustris (Salisb.) Schott 처녀고사리 KP256248

Dryopteridaceae 관중과

11 Arachniodes aristata (G. Forst.) Tindale 가는쇠고사리

12 Cyrtomium falcatum (L. f.) C. Presl 도깨비고비 NP582525

13 Dryopteris bissetiana (Baker) C. Chr. 산족제비고사리

14 Dryopteris chinensis (Baker) Koidz. 가는잎족제비고사리

15 Dryopteris erythrosora (D. C. Eaton) Kuntze 홍지네고사리 KP256150

16 Dryopteris lacera (Thunb.) Kuntze 비늘고사리 KP256154

17 Dryopteris pacifica (Nakai) Tagawa 큰족제비고사리

\section{Ginkgoaceae 은행나무과}

18 Ginkgo biloba L. 은행나무

19 Sciadopitys verticillata (Thunb.) Siebold \& Zucc. 금송

\section{Pinaceae 소나무과}

20 Pinus densiflora Siebold \& Zucc. 소나무 NP582538

21 Pinus rigida Mill. 리기다소나무

22 Pinus thunbergii Parl. 곰솔 KP255379

\section{Cupressaceae 측백나무과}

23 Chamaecyparis obtusa (Siebold \& Zucc.) Endl. 편백 KP255507

24 Chamaecyparis pisifera (Siebold \& Zucc.) Endl. 화백 NP582553

25 Cryptomeria japonica (Thunb. ex L. f.) D. Don 삼나무

26 Juniperus chinensis L. 향나무 NP582555 
Appendix 1. Continued.

\begin{tabular}{|c|c|c|c|c|c|c|c|}
\hline \multirow{2}{*}{ No. } & \multirow{2}{*}{$\begin{array}{c}\text { Taxa and representative voucher specimen number } \\
\text { (KP: KOSPVP0000-, KN: KONPVP0000-, NP: NIBRVP0000-) }\end{array}$} & \multicolumn{6}{|c|}{ Study } \\
\hline & & 1 & 2 & 3 & 4 & 5 & 6 \\
\hline 27 & Juniperus rigida Siebold \& Zucc. 노간주나무 KP255412 & $\mathrm{O}$ & & $\mathrm{O}$ & & $\mathrm{O}$ & $\mathrm{O}$ \\
\hline \multirow[t]{2}{*}{28} & Platycladus orientalis (L.) Franco 측백나무 NP582552 & & & & & & (0) \\
\hline & Lauraceae 녹나무과 & & & & & & \\
\hline 29 & Lindera glauca (Siebold \& Zucc.) Blume 감태나무 & & & $\mathrm{O}$ & & $\mathrm{O}$ & \\
\hline 30 & Lindera obtusiloba Blume 생강나무 & $\mathrm{O}$ & & & & & \\
\hline \multirow[t]{2}{*}{31} & Neolitsea sericea (Blume) Koidz. 참식나무 & & & & $\mathrm{O}$ & & \\
\hline & Chloranthaceae 홀아비꽃대과 & & & & & & \\
\hline 32 & Chloranthus fortunei (A. Gray) Sloms 옥녀꽃대 KP255426 & & & & & $\mathrm{O}$ & $\mathrm{O}$ \\
\hline \multirow[t]{2}{*}{33} & Chloranthus japonicus Siebold 홀아비꽃대 & $\mathrm{O}$ & & $\mathrm{O}$ & & $\mathrm{O}$ & \\
\hline & Saururaceae 삼백초과 & & & & & & \\
\hline \multirow[t]{2}{*}{34} & Houttuynia cordata Thunb. 약모밀 & & & & $\mathrm{O}$ & & \\
\hline & Ranunculaceae 미나리아재비과 & & & & & & \\
\hline 35 & Caltha palustris L. 동의나물 KP217375 & & & & $\mathrm{O}$ & $\mathrm{O}$ & $\mathrm{O}$ \\
\hline 36 & Clematis brachyura Maxim. 외대으아리 & & & & $\mathrm{O}$ & & \\
\hline 37 & Clematis terniflora DC. 참으아리 & & & & & $\mathrm{O}$ & \\
\hline 38 & Clematis terniflora DC. var. mandshurica (Rupr.) Ohwi 으아리 & & & & $\mathrm{O}$ & & \\
\hline 39 & Hepatica insularis Nakai 새끼노루귀 KP289427 & & & & & $\mathrm{O}$ & $\mathrm{O}$ \\
\hline 40 & Pulsatilla cernua (Thunb.) Bercht. \& J. Presl var. koreana (Yabe ex Nakai) Y. N. Lee 할미꽃 & $\mathrm{O}$ & & & & & \\
\hline 41 & Ranunculus cantoniensis DC. 털개구리미나리 & & & & $\mathrm{O}$ & & \\
\hline 42 & Ranunculus chinensis Bunge 젓가락나물 KP255573 & & & & & & $\mathrm{O}$ \\
\hline 43 & Ranunculus extorris Hance 개구리갓 & & & & $\mathrm{O}$ & & \\
\hline 44 & Ranunculus japonicus Thunb. 미나리아재비 KP255639 & & & & & & $\mathrm{O}$ \\
\hline 45 & Ranunculus sceleratus L. 개구리자리 KP255347 & & & & $\mathrm{O}$ & & $\mathrm{O}$ \\
\hline \multirow[t]{2}{*}{46} & Semiaquilegia adoxoides (DC.) Makino 개구리발톱 KP255453 & & & & & $\mathrm{O}$ & $\mathrm{O}$ \\
\hline & Menispermaceae 새모래덩굴과 & & & & & & \\
\hline \multirow[t]{2}{*}{47} & Cocculus trilobus (Thunb.) DC. 댕댕이덩굴 KP255688 & $\mathrm{O}$ & & $\mathrm{O}$ & $\mathrm{O}$ & $\mathrm{O}$ & $\mathrm{O}$ \\
\hline & Platanaceae 버즘나무과 & & & & & & \\
\hline \multirow[t]{2}{*}{48} & Platanus occidentalis L. 양버즘나무 & & & & (a) & & \\
\hline & Celtidaceae 팽나무과 & & & & & & \\
\hline \multirow[t]{2}{*}{49} & Celtis sinensis Pers. 팽나무 & $\mathrm{O}$ & & & $\mathrm{O}$ & & \\
\hline & Cannabinaceae 삼과 & & & & & & \\
\hline \multirow[t]{2}{*}{50} & Humulus japonicus Siebold \& Zucc. 환삼덩굴 KP256144 & & & & $\mathrm{O}$ & & $\mathrm{O}$ \\
\hline & Moraceae 뽛나무과 & & & & & & \\
\hline 51 & Broussonetia kazinoki Siebold 애기닥나무 KP256277 & & & & & & $\mathrm{O}$ \\
\hline 52 & Cudrania tricuspidata (Carriére) Bureau ex Lavallée 꾸지뽕나무 & & & $\mathrm{O}$ & & $\mathrm{O}$ & \\
\hline 53 & Ficus carica L. 무화과나무 & & & & (a) & & \\
\hline \multirow[t]{2}{*}{54} & Morus alba L. 뽕나무 KP255357 & & & & & & $\mathrm{O}$ \\
\hline & Urticaceae 쐐기풀과 & & & & & & \\
\hline 55 & Boehmeria nivea (L.) Gaudich. 모시풀 & & & & $\mathrm{O}$ & & \\
\hline
\end{tabular}


Appendix 1. Continued.

\begin{tabular}{|c|c|c|c|c|c|c|c|}
\hline \multirow{2}{*}{ No. } & \multirow{2}{*}{$\begin{array}{c}\text { Taxa and representative voucher specimen number } \\
\text { (KP: KOSPVP0000-, KN: KONPVP0000-, NP: NIBRVP0000-) }\end{array}$} & \multicolumn{6}{|c|}{ Study } \\
\hline & & 1 & 2 & 3 & 4 & 5 & 6 \\
\hline \multirow[t]{2}{*}{56} & Boehmeria tricuspis (Hance) Makino 거북꼬리 KP256280 & & & & & & $\mathrm{O}$ \\
\hline & Juglandaceae 가래나무과 & & & & & & \\
\hline \multirow[t]{2}{*}{57} & Platycarya strobilacea Siebold \& Zucc. 굴피나무 KP255364 & $\mathrm{O}$ & & $\mathrm{O}$ & $\mathrm{O}$ & $\mathrm{O}$ & $\mathrm{O}$ \\
\hline & Fagaceae 너도밤나무과 & & & & & & \\
\hline 58 & Castanea crenata Siebold \& Zucc. 밤나무 & & & (a) & & () & \\
\hline 59 & Quercus acutissima Carruth. 상수리나무 & $\mathrm{O}$ & & & & & \\
\hline 60 & Quercus aliena Blume 갈참나무 KP255361 & & & $\mathrm{O}$ & $\mathrm{O}$ & $\mathrm{O}$ & $\mathrm{O}$ \\
\hline 61 & Quercus dentata Thunb. 떡갈나무 KP255469 & $\mathrm{O}$ & & $\mathrm{O}$ & & $\mathrm{O}$ & $\mathrm{O}$ \\
\hline 62 & Quercus mongolica Fisch. ex Ledeb. 신갈나무 & $\mathrm{O}$ & & & & & \\
\hline 63 & Quercus myrsinifolia Blume 가시나무 & & & & $\mathrm{O}$ & & \\
\hline 64 & Quercus salicina Blume 참가시나무 NP582549 & & & & & & $\mathrm{O}$ \\
\hline 65 & Quercus serrata Murray 졸참나무 KP2 17370 & $\mathrm{O}$ & & $\mathrm{O}$ & $\mathrm{O}$ & $\mathrm{O}$ & $\mathrm{O}$ \\
\hline \multirow[t]{2}{*}{66} & Quercus variabilis Blume 굴참나무 & $\mathrm{O}$ & & $\mathrm{O}$ & & $\mathrm{O}$ & \\
\hline & Betulaceae 자작나무과 & & & & & & \\
\hline 67 & Alnus firma Siebold \& Zucc. 사방오리 KP255373 & (a) & & & (a) & (a) & (a) \\
\hline 68 & Alnus hirsuta Turcz. ex Rupr. 물오리나무 NP582545 & $\mathrm{O}$ & & & & & $\mathrm{O}$ \\
\hline 69 & Alnus japonica (Thunb.) Steud. 오리나무 & & & $\mathrm{O}$ & $\mathrm{O}$ & $\mathrm{O}$ & \\
\hline 70 & Carpinus heterophylla Fischer var. thunvergii Blume 개암나무 & $\mathrm{O}$ & & & & & \\
\hline \multirow[t]{2}{*}{71} & Carpinus turczaninowii Hance 소사나무 KP255456 & & & $\mathrm{O}$ & $\mathrm{O}$ & $\mathrm{O}$ & $\mathrm{O}$ \\
\hline & Phytolaccaceae 자리공과 & & & & & & \\
\hline \multirow[t]{2}{*}{72} & Phytolacca americana L. 미국자리공 KP255656 & & $\mathrm{O}$ & $\mathrm{O}$ & $\mathrm{O}$ & $\mathrm{O}$ & $\mathrm{O}$ \\
\hline & Chenopodiaceae 명아주과 & & & & & & \\
\hline 73 & Atriplex gmelinii C. A. Mey. 가는갯능쟁이 KN373425 & & & & $\mathrm{O}$ & & $\mathrm{O}$ \\
\hline 74 & Atriplex hastata L. 창명아주 & & & & $\mathrm{O}$ & & \\
\hline 75 & Chenopodium album L. 흰명아주 & & $\mathrm{O}$ & & $\mathrm{O}$ & & \\
\hline 76 & Chenopodium album L. var. centrorubrum Makino 명아주 & & & & $\mathrm{O}$ & & \\
\hline 77 & Chenopodium album L. var. stenophyllum Makino 가는명아주 & & & & $\mathrm{O}$ & & \\
\hline 78 & Chenopodium ficifolium Sm. 좀명아주 KP256199 & & & $\mathrm{O}$ & $\mathrm{O}$ & $\mathrm{O}$ & $\mathrm{O}$ \\
\hline 79 & Chenopodium glaucum L. 취명아주 KP255554 & & $\mathrm{O}$ & & & & $\mathrm{O}$ \\
\hline 80 & Kochia scoparia (L.) Schrad. 댑싸리 KP255670 & & & & (a) & & (a) \\
\hline 81 & Kochia scoparia (L.) Schrad. var. littorea Makino 갯댑싸리 & & & & $\mathrm{O}$ & & \\
\hline 82 & Salicornia europaea L. 퉁퉁마디 KP256218 & & & & $\mathrm{O}$ & & $\mathrm{O}$ \\
\hline 83 & Salsola komarovii Iljin 수송나물 KP255684 & & & & & & $\mathrm{O}$ \\
\hline 84 & Suaeda australis (R. Br.) Moq. 방석나물 KP256209 & & & & $\mathrm{O}$ & & $\mathrm{O}$ \\
\hline 85 & Suaeda glauca (Bunge) Bunge 나문재 KP255722 & & & & $\mathrm{O}$ & & $\mathrm{O}$ \\
\hline 86 & Suaeda japonica Makino 칠면초 KP256112 & & & & & & $\mathrm{O}$ \\
\hline \multirow[t]{2}{*}{87} & Suaeda maritima (L.) Dumort. 해홍나물 KP256088 & & & & $\mathrm{O}$ & & $\mathrm{O}$ \\
\hline & Amaranthaceae 비름과 & & & & & & \\
\hline 88 & Achyranthes fauriei H. Lèv. \& Vaniot 털쇠무릎 & & & & $\mathrm{O}$ & & \\
\hline
\end{tabular}


Appendix 1. Continued.

\begin{tabular}{|c|c|c|c|c|c|c|c|}
\hline \multirow{2}{*}{ No. } & \multirow{2}{*}{$\begin{array}{c}\text { Taxa and representative voucher specimen number } \\
\text { (KP: KOSPVP0000-, KN: KONPVP0000-, NP: NIBRVP0000-) }\end{array}$} & \multicolumn{6}{|c|}{ Study } \\
\hline & & 1 & 2 & 3 & 4 & 5 & 6 \\
\hline 89 & Achyranthes japonica (Miq.) Nakai 쇠무릎 KP256091 & $\mathrm{O}$ & & & & & $\mathrm{O}$ \\
\hline 90 & Amaranthus lividus L. 개비름 KP255662 & & & & & & $\mathrm{O}$ \\
\hline \multirow[t]{2}{*}{91} & Amaranthus viridis L. 청비름 & & $\mathrm{O}$ & & $\mathrm{O}$ & & \\
\hline & Portulacaceae 쇠비름과 & & & & & & \\
\hline \multirow[t]{2}{*}{92} & Portulaca oleracea L. 쇠비름 KN373186 & & & & & & $\mathrm{O}$ \\
\hline & Molluginaceae 석류풀과 & & & & & & \\
\hline \multirow[t]{2}{*}{93} & Mollugo pentaphylla L. 석류풀 KP255751 & & & & & & $\mathrm{O}$ \\
\hline & Caryophyllaceae 석죽과 & & & & & & \\
\hline 94 & Cerastium glomeratum Thuill. 양점나도나물 KP318927 & & $\mathrm{O}$ & & $\mathrm{O}$ & & $\mathrm{O}$ \\
\hline 95 & Cerastium holosteoides Fr. var. hallaisanense (Nakai) M. Mizush. 점나도나물 KP255408 & & & & & & $\mathrm{O}$ \\
\hline 96 & Dianthus chinensis L. 패랭이꽃 & $\mathrm{O}$ & & & & & \\
\hline 97 & Sagina japonica (Sw.) Ohwi 개미자리 KP255560 & & & & $\mathrm{O}$ & & $\mathrm{O}$ \\
\hline 98 & Sagina maxima A. Gray 큰개미자리 & & & & $\mathrm{O}$ & & \\
\hline 99 & Silene aprica Turcz. ex Fisch. \& C. A. Mey. var. oldhamiana (Miq.) C. Y. Wu 갯장구채 & $\mathrm{O}$ & & & & & \\
\hline 100 & Silene firma Siebold \& Zucc. 장구채 & $\mathrm{O}$ & & & & & \\
\hline 101 & Spergula arvensis L. 들개미자리 KP217372 & & & $\mathrm{O}$ & & $\mathrm{O}$ & $\mathrm{O}$ \\
\hline 102 & Spergularia marina (L.) Griseb. 갯개미자리 KP217355 & & & $\mathrm{O}$ & $\mathrm{O}$ & $\mathrm{O}$ & $\mathrm{O}$ \\
\hline 103 & Spergularia rubra J. Presl \& C. Presl 유럽개미자리 & & $\mathrm{O}$ & & $\mathrm{O}$ & & \\
\hline 104 & Stellaria aquatica (L.) Scop. 쇠별꽃 KP256156 & & & & $\mathrm{O}$ & & $\mathrm{O}$ \\
\hline \multirow[t]{2}{*}{105} & Stellaria media (L.) Vill. 별꽃 KP255611 & & & $\mathrm{O}$ & $\mathrm{O}$ & $\mathrm{O}$ & $\mathrm{O}$ \\
\hline & Polygonaceae 마디풀과 & & & & & & \\
\hline 106 & Fallopia convolvulus (L.) Á. Löve 나도닭의덩굴 & & $\mathrm{O}$ & & & & \\
\hline 107 & Polygonum aviculare L. 마디풀 KP256108 & & & & $\mathrm{O}$ & $\mathrm{O}$ & $\mathrm{O}$ \\
\hline 108 & Polygonum fusco-ochreatum Kom. 큰옥매듭풀 KP319812 & & & & & & $\mathrm{O}$ \\
\hline 109 & Polygonum japonicum Meisn. 흰꽃여뀌 KP255712 & & & & & & $\mathrm{O}$ \\
\hline 110 & Polygonum lapathifolium L. 흰여뀌 KP255663 & & & & $\mathrm{O}$ & $\mathrm{O}$ & $\mathrm{O}$ \\
\hline 111 & Polygonum longisetum Bruijn 개여뀌 KP256100 & $\mathrm{O}$ & & & $\mathrm{O}$ & & $\mathrm{O}$ \\
\hline 112 & Polygonum perfoliatum L. 며느리배꼽 KP256145 & & & & $\mathrm{O}$ & & $\mathrm{O}$ \\
\hline 113 & Polygonum polyneuron Franch. \& Sav. 갯마디풀 & & & & $\mathrm{O}$ & & \\
\hline 114 & Polygonum sagittatum L. var. sieboldii (Meisn.) Maxim 미꾸리낚시 KP256120 & & & & & $\mathrm{O}$ & $\mathrm{O}$ \\
\hline 115 & Polygonum thunbergii Siebold \& Zucc. 고마리 KP256137 & & & & & & $\mathrm{O}$ \\
\hline 116 & Rumex acetosa L. 수영 KP217358 & & & & & & $\mathrm{O}$ \\
\hline 117 & Rumex acetosella L. 애기수영 KP255513 & & $\mathrm{O}$ & $\mathrm{O}$ & & $\mathrm{O}$ & $\mathrm{O}$ \\
\hline 118 & Rumex conglomeratus Murray 묵밭소리쟁이 KP255520 & & & & & & $\mathrm{O}$ \\
\hline 119 & Rumex crispus L. 소리쟁이 KP290394 & $\mathrm{O}$ & $\mathrm{O}$ & $\mathrm{O}$ & $\mathrm{O}$ & $\mathrm{O}$ & $\mathrm{O}$ \\
\hline 120 & Rumex japonicus Houtt. 참소리쟁이 KP255695 & & & & $\mathrm{O}$ & & $\mathrm{O}$ \\
\hline 121 & Rumex nipponicus Franch. \& Sav. 좀소리쟁이 KP290422 & & & & & & $\mathrm{O}$ \\
\hline 122 & Rumex obtusifolius L. 돌소리쟁이 & & $\mathrm{O}$ & & $\mathrm{O}$ & & \\
\hline & Plumbaginaceae 갯질경이과 & & & & & & \\
\hline
\end{tabular}


Appendix 1. Continued.

\begin{tabular}{|c|c|c|c|c|c|c|c|}
\hline \multirow{2}{*}{ No. } & \multirow{2}{*}{$\begin{array}{c}\text { Taxa and representative voucher specimen number } \\
\text { (KP: KOSPVP0000-, KN: KONPVP0000-, NP: NIBRVP0000-) }\end{array}$} & \multicolumn{6}{|c|}{ Study } \\
\hline & & 1 & 2 & 3 & 4 & 5 & 6 \\
\hline \multirow[t]{2}{*}{123} & Limonium tetragonum (Thunb.) Bullock 갯길경 KP255703 & & & & & $\mathrm{O}$ & $\mathrm{O}$ \\
\hline & Theaceae 차나무과 & & & & & & \\
\hline 124 & Camellia japonica L. 동백나무 NP582533 & $\mathrm{O}$ & & & & $\mathrm{O}$ & $\mathrm{O}$ \\
\hline 125 & Camellia sasanqua Thunb. 애기동백 NP582548 & & & & & & (a) \\
\hline \multirow[t]{2}{*}{126} & Eurya japonica Thunb. 사스레피나무 KP217371 & $\mathrm{O}$ & & $\mathrm{O}$ & $\mathrm{O}$ & $\mathrm{O}$ & $\mathrm{O}$ \\
\hline & Guttiferae 물레나물과 & & & & & & \\
\hline 127 & Hypericum erectum Thunb. 고추나물 KP255740 & $\mathrm{O}$ & & & $\mathrm{O}$ & $\mathrm{O}$ & $\mathrm{O}$ \\
\hline 128 & Hypericum japonicum Thunb. 애기고추나물 KP256119 & & & & $\mathrm{O}$ & $\mathrm{O}$ & $\mathrm{O}$ \\
\hline \multirow[t]{2}{*}{129} & Hypericum laxum (Blume) Koidz. 좀고추나물 KP255763 & $\mathrm{O}$ & & & $\mathrm{O}$ & & $\mathrm{O}$ \\
\hline & Tiliaceae 피나무과 & & & & & & \\
\hline 130 & Corchoropsis tomentosa (Thunb.) Makino 수까치깨 KP256162 & $\mathrm{O}$ & & & & & $\mathrm{O}$ \\
\hline \multirow[t]{2}{*}{131} & Grewia parviflora Bunge 장구밥나무 KP255680 & & & & & & $\mathrm{O}$ \\
\hline & Sterculiaceae 벽오동과 & & & & & & \\
\hline \multirow[t]{2}{*}{132} & Firmiana simplex (L.) W. Wight 벽오동 & & & & (a) & & \\
\hline & Malvaceae 아욱과 & & & & & & \\
\hline 133 & Abutilon theophrasti Medik. 어저귀 & & $\mathrm{O}$ & & & & \\
\hline 134 & Malva neglecta Wallr. 난쟁이아욱 NP582529 & & & & & & $\mathrm{O}$ \\
\hline \multirow[t]{2}{*}{135} & Malva sylvestris L. var. mauritiana (L.) Boiss. 당아욱 & & $\mathrm{O}$ & & & & \\
\hline & Flacourtiaceae 산유자나무과 & & & & & & \\
\hline \multirow[t]{2}{*}{136} & Idesia polycarpa Maxim. 이나무 KP255626 & & & & & & $\mathrm{O}$ \\
\hline & Violaceae 제비꽃과 & & & & & & \\
\hline 137 & Viola acuminata Ledeb. 졸방제비꽃 KP255535 & & & & & $\mathrm{O}$ & $\mathrm{O}$ \\
\hline 138 & Viola albida Palib. var. chaerophylloides (Regel) F. Maek. 남산제비꽃 & $\mathrm{O}$ & & & & $\mathrm{O}$ & \\
\hline 139 & Viola grypoceras A. Gray 낚시제비꽃 KP255447 & & & $\mathrm{O}$ & & $\mathrm{O}$ & $\mathrm{O}$ \\
\hline 140 & Viola japonica Langsd. ex Ging. 왜제비꽃 NP582536 & & & & & $\mathrm{O}$ & $\mathrm{O}$ \\
\hline 141 & Viola lactiflora Nakai 흰젖제비꽃 KP319810 & & & $\mathrm{O}$ & & & $\mathrm{O}$ \\
\hline 142 & Viola mandshurica W. Becker 제비꽃 KP255370 & $\mathrm{O}$ & & & $\mathrm{O}$ & $\mathrm{O}$ & $\mathrm{O}$ \\
\hline 143 & Viola patrinii DC. ex Ging. 흰제비꽃 & & & & & $\mathrm{O}$ & \\
\hline 144 & Viola rossii Hemsl. 고깔제비꽃 & & & & & $\mathrm{O}$ & \\
\hline \multirow[t]{2}{*}{145} & Viola yedoensis Makino 호제비꽃 & & & & $\mathrm{O}$ & & \\
\hline & Cucurbitaceae 박과 & & & & & & \\
\hline 146 & Actinostemma lobatum Maxim. 뚜껑덩굴 & & & & $\mathrm{O}$ & & \\
\hline \multirow[t]{2}{*}{147} & Trichosanthes kirilowii Maxim. var. japonica (Miq.) Kitam. 노랑하늘타리 KP255761 & & & & & & $\mathrm{O}$ \\
\hline & Salicaceae 버드나무과 & & & & & & \\
\hline 148 & Populus $\times$ tomentiglandulosa T. B. Lee 은사시나무 & (a) & & & (a) & & \\
\hline \multirow[t]{2}{*}{149} & Salix koreensis Andersson 버드나무 KP217379 & & & & $\mathrm{O}$ & & $\mathrm{O}$ \\
\hline & Brassicaceae 십자화과 & & & & & & \\
\hline 150 & Arabis glabra (L.) Bernh. 장대나물 & & & & & $\mathrm{O}$ & \\
\hline 151 & Brassica juncea (L.) Czern. 갓 KP255342 & & $\mathrm{O}$ & & $\mathrm{O}$ & $\mathrm{O}$ & $\mathrm{O}$ \\
\hline
\end{tabular}


Appendix 1. Continued.

\begin{tabular}{|c|c|c|c|c|c|c|c|}
\hline \multirow{2}{*}{ No. } & \multirow{2}{*}{$\begin{array}{l}\text { Taxa and representative voucher specimen number } \\
\text { (KP: KOSPVP0000-, KN: KONPVP0000-, NP: NIBRVP0000-) }\end{array}$} & \multicolumn{6}{|c|}{ Study } \\
\hline & & 1 & 2 & 3 & 4 & 5 & 6 \\
\hline 152 & Capsella bursa-pastoris (L.) Medik. 냉이 KP255341 & & & & $\mathrm{O}$ & $\mathrm{O}$ & $\mathrm{O}$ \\
\hline 153 & Cardamine fallax (O. E. Schulz) Nakai 좁쌀냉이 NP582559 & & & & & $\mathrm{O}$ & $\mathrm{O}$ \\
\hline 154 & Cardamine flexuosa With. 황새냉이 KP255430 & & & $\mathrm{O}$ & $\mathrm{O}$ & $\mathrm{O}$ & $\mathrm{O}$ \\
\hline 155 & Cardamine impatiens L. 싸리냉이 KP255406 & & & & & & $\mathrm{O}$ \\
\hline 156 & Lepidium apetalum Willd. 다닥냉이 KP255544 & & $\mathrm{O}$ & & & & $\mathrm{O}$ \\
\hline 157 & Lepidium virginicum L. 콩다닥냉이 KP290396 & & $\mathrm{O}$ & $\mathrm{O}$ & $\mathrm{O}$ & $\mathrm{O}$ & $\mathrm{O}$ \\
\hline 158 & Rorippa indica (L.) Hiern 개갓냉이 KP255387 & & & & $\mathrm{O}$ & & $\mathrm{O}$ \\
\hline 159 & Rorippa palustris (L.) Besser 속속이풀 KP255606 & & & & $\mathrm{O}$ & & $\mathrm{O}$ \\
\hline \multirow[t]{2}{*}{160} & Thlaspi arvense L. 말냉이 & & $\mathrm{O}$ & & & & \\
\hline & Ericaceae 진달래과 & & & & & & \\
\hline 161 & Rhododendron mucronulatum Turcz. 진달래 NP582523 & $\mathrm{O}$ & & $\mathrm{O}$ & $\mathrm{O}$ & $\mathrm{O}$ & $\mathrm{O}$ \\
\hline 162 & Rhododendron yedoense Maxim. ex Regel for. poukhanense (H. Lév.) M. Sugim. 산철쭉 KP255365 & & & $\mathrm{O}$ & $\mathrm{O}$ & $\mathrm{O}$ & $\mathrm{O}$ \\
\hline 163 & Vaccinium bracteatum Thunb. 모새나무 KP255637 & $\mathrm{O}$ & & & $\mathrm{O}$ & $\mathrm{O}$ & $\mathrm{O}$ \\
\hline \multirow[t]{2}{*}{164} & Vaccinium oldhamii Miq. 정금나무 KP217365 & $\mathrm{O}$ & & $\mathrm{O}$ & $\mathrm{O}$ & $\mathrm{O}$ & $\mathrm{O}$ \\
\hline & Pyrolaceae 노루발과 & & & & & & \\
\hline \multirow[t]{2}{*}{165} & Pyrola japonica Klenze ex Alef. 노루발 NP582550 & $\mathrm{O}$ & & & $\mathrm{O}$ & $\mathrm{O}$ & $\mathrm{O}$ \\
\hline & Ebenaceae 감나무과 & & & & & & \\
\hline \multirow[t]{2}{*}{166} & Diospyros kaki Thunb. 감나무 & (a) & & & & & \\
\hline & Styracaceae 때죽나무과 & & & & & & \\
\hline \multirow[t]{2}{*}{167} & Styrax japonicus Siebold \& Zucc. 때죽나무 KP255557 & & & & & & $\mathrm{O}$ \\
\hline & Symplocaceae 노린재나무과 & & & & & & \\
\hline 168 & Symplocos sawafutagi Nagam. 노린재나무 KP255514 & $\mathrm{O}$ & & & & $\mathrm{O}$ & $\mathrm{O}$ \\
\hline \multirow[t]{2}{*}{169} & Symplocos tanakana Nakai 검노린재 KP217364 & & & & $\mathrm{O}$ & $\mathrm{O}$ & $\mathrm{O}$ \\
\hline & Primulaceae 앵초과 & & & & & & \\
\hline 170 & Androsace filiformis Retz. 애기봄맞이 KP256133 & & & & & & $\mathrm{O}$ \\
\hline 171 & Lysimachia barystachys Bunge 까치수염 & & & & & $\mathrm{O}$ & \\
\hline 172 & Lysimachia clethroides Duby 큰까치수염 KP255632 & $\mathrm{O}$ & & $\mathrm{O}$ & & $\mathrm{O}$ & $\mathrm{O}$ \\
\hline 173 & Lysimachia japonica Thunb. 좀가지풀 KP255533 & & & & $\mathrm{O}$ & & $\mathrm{O}$ \\
\hline \multirow[t]{2}{*}{174} & Lysimachia mauritiana Lam. 갯까치수염 KP255701 & & & & $\mathrm{O}$ & & $\mathrm{O}$ \\
\hline & Crassulaceae 돌나물과 & & & & & & \\
\hline 175 & Sedum bulbiferum Makino 말똥비름 & & & & $\mathrm{O}$ & & \\
\hline \multirow[t]{2}{*}{176} & Sedum oryzifolium Makino 땅채송화 & & & $\mathrm{O}$ & & $\mathrm{O}$ & \\
\hline & Parnassiaceae 물매화과 & & & & & & \\
\hline \multirow[t]{2}{*}{177} & Parnassia palustris L. var. multiseta Ledeb. 물매화 KP255770 & $\mathrm{O}$ & & & $\mathrm{O}$ & & $\mathrm{O}$ \\
\hline & Rosaceae 장미과 & & & & & & \\
\hline 178 & Agrimonia pilosa Ledeb. 짚신나물 KP255738 & & & & & & $\mathrm{O}$ \\
\hline 179 & Aria alnifolia (Siebold \& Zucc.) Decne. 팥배나무 KP255432 & & & $\mathrm{O}$ & $\mathrm{O}$ & $\mathrm{O}$ & $\mathrm{O}$ \\
\hline 180 & Duchesnea chrysantha (Zoll. \& Moritzi) Miq. 뱀딸기 & & & & $\mathrm{O}$ & $\mathrm{O}$ & \\
\hline 181 & Geum japonicum Thunb. 뱀무 & $\mathrm{O}$ & & & & & \\
\hline
\end{tabular}


Appendix 1. Continued.

\begin{tabular}{|c|c|c|c|c|c|c|c|}
\hline \multirow{2}{*}{ No. } & \multirow{2}{*}{$\begin{array}{c}\text { Taxa and representative voucher specimen number } \\
\text { (KP: KOSPVP0000-, KN: KONPVP0000-, NP: NIBRVP0000-) }\end{array}$} & \multicolumn{6}{|c|}{ Study } \\
\hline & & 1 & 2 & 3 & 4 & 5 & 6 \\
\hline 182 & Potentilla chinensis Ser. 딱지꽃 & & & & $\mathrm{O}$ & & \\
\hline 183 & Potentilla discolor Bunge 솜양지꽃 KP217369 & $\mathrm{O}$ & & $\mathrm{O}$ & $\mathrm{O}$ & $\mathrm{O}$ & $\mathrm{O}$ \\
\hline 184 & Potentilla fragarioides L. 양지꽃 NP582541 & & & & & $\mathrm{O}$ & $\mathrm{O}$ \\
\hline 185 & Potentilla freyniana Bornm. 세잎양지 꽃 KP255380 & & & & & $\mathrm{O}$ & $\mathrm{O}$ \\
\hline 186 & Potentilla supina L. 개소시랑개비 & & $\mathrm{O}$ & & & & \\
\hline 187 & Pourthiaea villosa (Thunb.) Decne. 윤노리나무 & & & & & $\mathrm{O}$ & \\
\hline 188 & Prunus jamasakura Siebold ex Koidz. 벚나무 KP255423 & & & & & & $\mathrm{O}$ \\
\hline 189 & Prunus mume (Siebold) Siebold \& Zucc. 매실나무 NP582534 & & & & & & (a) \\
\hline 190 & Prunus sargentii Rehder 산벚나무 KP255455 & $\mathrm{O}$ & & & & & $\mathrm{O}$ \\
\hline 191 & Prunus serrulata Lindl. var. pubescens (Makino) Nakai 잔털벚나무 & & & $\mathrm{O}$ & $\mathrm{O}$ & $\mathrm{O}$ & \\
\hline 192 & Pyrus calleryana Decne. var. fauriei (C. K. Schneid.) Rehder 콩배나무 KP255378 & & & & $\mathrm{O}$ & $\mathrm{O}$ & $\mathrm{O}$ \\
\hline 193 & Pyrus pyrifolia (Burm. f.) Nakai 돌배나무 KP255559 & & & & & & $\mathrm{O}$ \\
\hline 194 & Rosa multiflora Thunb. 찔레나무 KP255360 & $\mathrm{O}$ & & $\mathrm{O}$ & $\mathrm{O}$ & $\mathrm{O}$ & $\mathrm{O}$ \\
\hline 195 & Rosa rugosa Thunb. 해당화 KP255584 & $\mathrm{O}$ & & & & & $\mathrm{O}$ \\
\hline 196 & Rosa wichuraiana Crép. ex Déségl. 돌가시나무 KP256121 & $\mathrm{O}$ & & $\mathrm{O}$ & $\mathrm{O}$ & $\mathrm{O}$ & $\mathrm{O}$ \\
\hline 197 & Rubus hirsutus Thunb. 장딸기 KP255445 & & & & & & $\mathrm{O}$ \\
\hline 198 & Rubus parvifolius L. 멍석딸기 KP255367 & $\mathrm{O}$ & & $\mathrm{O}$ & $\mathrm{O}$ & $\mathrm{O}$ & $\mathrm{O}$ \\
\hline 199 & Sanguisorba officinalis L. 오이풀 KP255731 & $\mathrm{O}$ & & & $\mathrm{O}$ & $\mathrm{O}$ & $\mathrm{O}$ \\
\hline & Fabaceae 콩과 & & & & & & \\
\hline 200 & Aeschynomene indica L. 자귀풀 KP255706 & & & & $\mathrm{O}$ & $\mathrm{O}$ & $\mathrm{O}$ \\
\hline 201 & Albizia kalkora (Roxb.) Prain 왕자귀나무 KP255759 & $\mathrm{O}$ & & $\mathrm{O}$ & $\mathrm{O}$ & $\mathrm{O}$ & $\mathrm{O}$ \\
\hline 202 & Albizzia julibrissin Durazz. 자귀나무 KP256206 & & & $\mathrm{O}$ & $\mathrm{O}$ & $\mathrm{O}$ & $\mathrm{O}$ \\
\hline 203 & Amorpha fruticosa L. 족제비싸리 KP255622 & & $\mathrm{O}$ & & & & \\
\hline 204 & Amphicarpaea bracteata (L.) Fernald subsp. edgeworthii (Benth.) H. Ohashi 새콩 KP256159 & & & & & & $\mathrm{O}$ \\
\hline 205 & Chamaecrista nomame (Siebold) H. Ohashi 차풀 KP256111 & & & & & $\mathrm{O}$ & $\mathrm{O}$ \\
\hline 206 & Dunbaria villosa (Thunb.) Makino 여우팥 KP255739 & & & & $\mathrm{O}$ & $\mathrm{O}$ & $\mathrm{O}$ \\
\hline 207 & Glycine soja Siebold \& Zucc. 돌콩 & & & & $\mathrm{O}$ & $\mathrm{O}$ & \\
\hline 208 & Indigofera bungeana Walp. 큰낭아초 KN373182 & & & & & & $\mathrm{O}$ \\
\hline 209 & Indigofera kirilowii Maxim. ex Palib. 땅비싸리 & $\mathrm{O}$ & & & $\mathrm{O}$ & & \\
\hline 210 & Indigofera koreana Ohwi 좀땅비싸리 KP217362 & & & $\mathrm{O}$ & $\mathrm{O}$ & $\mathrm{O}$ & $\mathrm{O}$ \\
\hline & Kummerowia stipulacea (Maxim.) Makino 둥근매듭풀 KP255696 & & & & $\mathrm{O}$ & & $\mathrm{O}$ \\
\hline 212 & Kummerowia striata (Thunb.) Schindl. 매듭풀 KP255692 & $\mathrm{O}$ & & & $\mathrm{O}$ & & $\mathrm{O}$ \\
\hline 213 & Lathyrus japonicus Willd. 갯완두 KP255578 & & & & & & $\mathrm{O}$ \\
\hline & Lespedeza bicolor Turcz. 싸리 KP255687 & & & & $\mathrm{O}$ & $\mathrm{O}$ & $\mathrm{O}$ \\
\hline 215 & Lespedeza cuneata (Dum. Cours.) G. Don. 비수리 KP255698 & $\mathrm{O}$ & & & $\mathrm{O}$ & $\mathrm{O}$ & $\mathrm{O}$ \\
\hline 216 & Lespedeza cyrtobotrya Miq. 참싸리 KP255635 & $\mathrm{O}$ & & & & & $\mathrm{O}$ \\
\hline & Lespedeza maritima Nakai 해변싸리 KP255631 & $\mathrm{O}$ & & & & & $\mathrm{O}$ \\
\hline 218 & Lespedeza maximowiczii C. K. Schneid. 조록싸리 KP255707 & $\mathrm{O}$ & & $\mathrm{O}$ & & $\mathrm{O}$ & $\mathrm{O}$ \\
\hline & Lespedeza maximowiczii C. K. Schneider var. tomentella Nakai 털조록싸리 KP291304 & & & & & & $\mathrm{O}$ \\
\hline
\end{tabular}


Appendix 1. Continued.

\begin{tabular}{|c|c|c|c|c|c|c|c|}
\hline \multirow{2}{*}{ No. } & \multirow{2}{*}{$\begin{array}{l}\text { Taxa and representative voucher specimen number } \\
\text { (KP: KOSPVP0000-, KN: KONPVP0000-, NP: NIBRVP0000-) }\end{array}$} & \multicolumn{6}{|c|}{ Study } \\
\hline & & 1 & 2 & 3 & 4 & 5 & 6 \\
\hline 220 & Lespedeza pilosa (Thunb.) Siebold \& Zucc. 괭이싸리 & $\mathrm{O}$ & & & $\mathrm{O}$ & $\mathrm{O}$ & \\
\hline 221 & Lespedeza tomentosa (Thunb.) Siebold ex Maxim. 개싸리 & & & & $\mathrm{O}$ & & \\
\hline 222 & Lespedeza virgata (Thunb.) DC. 좀싸리 KP255668 & & & & $\mathrm{O}$ & $\mathrm{O}$ & $\mathrm{O}$ \\
\hline 223 & Lotus corniculatus L. var. japonicus Regel 벌노랑이 KP217359 & & & & $\mathrm{O}$ & & $\mathrm{O}$ \\
\hline 224 & Medicago lupulina L. 잔개자리 & & $\mathrm{O}$ & & $\mathrm{O}$ & & \\
\hline 225 & Medicago polymorpha L. 개자리 KP255348 & & & & $\mathrm{O}$ & & $\mathrm{O}$ \\
\hline 226 & Melilotus suaveolens Ledeb. 전동싸리 & & $\mathrm{O}$ & & & & \\
\hline 227 & Pueraria lobata (Willd.) Ohwi 칡 & & & & $\mathrm{O}$ & & \\
\hline 228 & Rhynchosia volubilis Lour. 여우콩 & & & & & $\mathrm{O}$ & \\
\hline 229 & Robinia pseudoacacia L. 아까시나무 KP255366 & $\mathrm{O}$ & $\mathrm{O}$ & & $\mathrm{O}$ & & $\mathrm{O}$ \\
\hline 230 & Sophora flavescens Aiton 고삼 & $\mathrm{O}$ & & $\mathrm{O}$ & $\mathrm{O}$ & $\mathrm{O}$ & \\
\hline 231 & Trifolium repens L. 토끼풀 KP255356 & $\mathrm{O}$ & $\mathrm{O}$ & & $\mathrm{O}$ & & $\mathrm{O}$ \\
\hline 232 & Vicia amoena Fisch. 갈퀴나물 & & & & & $\mathrm{O}$ & \\
\hline 233 & Vicia angustifolia L. ex Reichard 가는살갈퀴 KP255340 & & & & & & $\mathrm{O}$ \\
\hline 234 & Vicia angustifolia L. ex Reichard var. segetilis (Thuill) W. D. J. Koch 살갈퀴 & & & & $\mathrm{O}$ & $\mathrm{O}$ & \\
\hline 235 & Vicia cracca L. 등갈퀴나물 KP255375 & & & & & & $\mathrm{O}$ \\
\hline 236 & Vicia hirsuta (L.) Gray 새완두 KP255515 & & & & & & $\mathrm{O}$ \\
\hline 237 & Vicia tetrasperma (L.) Schreb. 얼치기완두 KP255344 & & & & $\mathrm{O}$ & $\mathrm{O}$ & $\mathrm{O}$ \\
\hline 238 & Vicia unijuga A. Braun 나비나물 & & & & & $\mathrm{O}$ & \\
\hline 239 & Vicia villosa Roth 벳지 KP255569 & & & & $\mathrm{O}$ & & $\mathrm{O}$ \\
\hline 240 & Vigna angularis (Willd.) Ohwi \& H. Ohashi var. nipponensis (Ohwi) Ohwi \& H. Ohashi 새팥 KP256129 & & & & $\mathrm{O}$ & & $\mathrm{O}$ \\
\hline 241 & Vigna vexillata (L.) A. Rich. var. tsusimensis Matsum. 돌동부 KP256233 & & & & & & $\mathrm{O}$ \\
\hline \multirow[t]{2}{*}{242} & Wisteria japonica Siebold \& Zucc. 애기등 KP255762 & & & & $\mathrm{O}$ & & $\mathrm{O}$ \\
\hline & Elaeagnaceae 보리수나무과 & & & & & & \\
\hline \multirow[t]{2}{*}{243} & Elaeagnus umbellata Thunb. 보리수나무 KP255382 & $\mathrm{O}$ & & & $\mathrm{O}$ & $\mathrm{O}$ & $\mathrm{O}$ \\
\hline & Haloragaceae 개미탑과 & & & & & & \\
\hline \multirow[t]{2}{*}{244} & Haloragis micrantha (Thunb.) R. Br. ex Siebold \& Zucc. 개미탑 KP255425 & $\mathrm{O}$ & & & $\mathrm{O}$ & $\mathrm{O}$ & $\mathrm{O}$ \\
\hline & Lythraceae 부처꽃과 & & & & & & \\
\hline 245 & Lagerstroemia indica L. 배롱나무 NP582556 & & & & & & (a) \\
\hline \multirow[t]{2}{*}{246} & Rotala indica (Willd.) Koehne 마디꽃 & & & & $\mathrm{O}$ & & \\
\hline & Trapaceae 마름과 & & & & & & \\
\hline \multirow[t]{2}{*}{247} & Trapa japonica Flerow 마름 KP255650 & & & & $\mathrm{O}$ & & $\mathrm{O}$ \\
\hline & Onagraceae 바늘꽃과 & & & & & & \\
\hline 248 & Epilobium pyrricholophum Franch. \& Sav. 바늘꽃 & & & & & $\mathrm{O}$ & \\
\hline 249 & Ludwigia epilobioides Maxim. 여뀌바늘 & & & & $\mathrm{O}$ & & \\
\hline 250 & Oenothera biennis L. 달맞이꽃 KP255664 & $\mathrm{O}$ & $\mathrm{O}$ & & $\mathrm{O}$ & & $\mathrm{O}$ \\
\hline \multirow[t]{2}{*}{251} & Oenothera glazioviana Micheli 큰달맞이꽃 KP255694 & & & & & & $\mathrm{O}$ \\
\hline & Cornaceae 충충나무과 & & & & & & \\
\hline 252 & Cornus officinalis Siebold \& Zucc. 산수유 NP582542 & & & & & & (a) \\
\hline
\end{tabular}


Appendix 1. Continued.

\begin{tabular}{llllllll}
\hline \hline \multirow{2}{*}{ No. } & Taxa and representative voucher specimen number & & \multicolumn{3}{c}{ Study } & \multicolumn{3}{c}{} \\
\cline { 3 - 7 } & (KP: KOSPVP0000-, KN: KONPVP0000-, NP: NIBRVP0000-) & 1 & 2 & 3 & 4 & 5
\end{tabular}

\section{Santalaceae 단향과}

253 Thesium chinense Turcz. 제비꿀 KP255524

$\mathrm{O}$

Celastraceae 노박덩굴과

254 Celastrus orbiculatus Thunb. 노박덩굴 KP255528

255 Euonymus japonicus Thunb. 사철나무 KP255349

256 Euonymus sachalinensis (F. Schmidt) Maxim. 회나무

257 Tripterygium regelii Sprague \& Takeda 미역줄나무

Aquifoliaceae 감탕나무과

258 Ilex crenata Thunb. 꽝꽝나무 KP290404

\section{Euphorbiaceae 대극과}

259 Acalypha australis L. 깨풀 KP255676

260 Euphorbia esula L. 흰대극

261 Euphorbia helioscopia L. 등대풀 KP255561

262 Euphorbia sieboldiana C. Morren \& Decne. 개감수 KP255512

263 Euphorbia supina Raf. 애기땅빈대 KP256229

264 Mallotus japonicus (L. f.) Müll. Arg. 예덕나무 KP255633

265 Phyllanthus ussuriensis Rupr. \& Maxim. 여우주머니 KP256163

\section{Vitaceae 포도과}

266 Ampelopsis brevipedunculata (Maxim.) Trautv. 개머루 KP255657

267 Parthenocissus tricuspidata (Siebold \& Zucc.) Planch. 담쟁이덩굴

268 Vitis amurensis Rupr. 왕머루

269 Vitis ficifolia Bunge var. sinuata (Regel) H. Hara 까마귀머루 KP255682

270 Vitis flexuosa Thunb. 새머루 KP255689

\section{Polygalaceae 원지과}

271 Polygala japonica Houtt. 애기풀 KP255424

272 Salomonia oblongifolia DC. 병아리다리 KP319784

\section{Staphyleaceae 고추나무과}

273 Euscaphis japonica (Thunb.) Kanitz 말오줌때 KP255381

274 Staphylea bumalda DC. 고추나무 KP255744

\section{Anacardiaceae 옻나무과}

275 Rhus javanica L. 붉나무 KP255730

276 Toxicodendron sylvestre (Siebold \& Zucc.) Kuntze 산검양옻나무 KP255562

277 Toxicodendron trichocarpum (Miq.) Kuntze 개옻나무 KP255386

\section{Meliaceae 멀구슬나무과}

278 Melia azedarach L. 멀구슬나무 KP255567

\section{Rutaceae 운향과}

279 Orixa japonica Thunb. 상산

$\mathrm{O}$

$\mathrm{O}$

280 Poncirus trifoliata (L.) Raf. 탱자나무 KP255437

281 Zanthoxylum planispinum Siebold \& Zucc. 개산초

$$
\mathrm{O} \quad \mathrm{O}
$$$$
\mathrm{O} \quad \mathrm{O} \quad \mathrm{O}
$$

$\mathrm{O}$

$\begin{array}{llll}\mathrm{O} & \mathrm{O} & \mathrm{O} & \mathrm{O}\end{array}$

$\mathrm{O}$

O O O

$\mathrm{O}$

$\mathrm{O}$

$\mathrm{O}$

O O O O

$\mathrm{O}$

$\begin{array}{llll}\mathrm{O} & \mathrm{O} & \mathrm{O} & \mathrm{O}\end{array}$

$\mathrm{O}$

$\mathrm{O}$

O

$\mathrm{O}$

$\mathrm{O}$ 
Appendix 1. Continued.

\begin{tabular}{|c|c|c|c|c|c|c|c|}
\hline \multirow{2}{*}{ No. } & \multirow{2}{*}{$\begin{array}{c}\text { Taxa and representative voucher specimen number } \\
\text { (KP: KOSPVP0000-, KN: KONPVP0000-, NP: NIBRVP0000-) }\end{array}$} & \multicolumn{6}{|c|}{ Study } \\
\hline & & 1 & 2 & 3 & 4 & 5 & 6 \\
\hline \multirow[t]{2}{*}{282} & Zanthoxylum schinifolium Siebold \& Zucc. 산초나무 KP255720 & $\mathrm{O}$ & & & $\mathrm{O}$ & & $\mathrm{O}$ \\
\hline & Oxalidaceae 괭이밥과 & & & & & & \\
\hline 283 & Oxalis articulata Sabigny 덩이괭이밥 & & $\mathrm{O}$ & & & & \\
\hline 284 & Oxalis corniculata L. 괭이밥 KP255338 & $\mathrm{O}$ & & $\mathrm{O}$ & $\mathrm{O}$ & $\mathrm{O}$ & $\mathrm{O}$ \\
\hline \multirow[t]{2}{*}{285} & Oxalis stricta L. 선괭이밥 & & & & $\mathrm{O}$ & & \\
\hline & Balsaminaceae 봉선화과 & & & & & & \\
\hline \multirow[t]{2}{*}{286} & Impatiens noli-tangere L. 노랑물봉선 & $\mathrm{O}$ & & & & & \\
\hline & Araliaceae 두릅나무과 & & & & & & \\
\hline 287 & Aralia elata (Miq.) Seem. 두릅나무 & & & & $\mathrm{O}$ & & \\
\hline 288 & Fatsia japonica (Thunb.) Decne. \& Planch. 팔손이 NP582527 & & & & & & $\mathrm{O}$ \\
\hline \multirow[t]{2}{*}{289} & Hedera rhombea (Miq.) Bean 송악 NP582557 & & & & & & $\mathrm{O}$ \\
\hline & Apiaceae 미나리과 & & & & & & \\
\hline 290 & Bupleurum falcatum L. 시호 KP255764 & $\mathrm{O}$ & & & $\mathrm{O}$ & & $\mathrm{O}$ \\
\hline 291 & Caucalis scabra (DC.) Makino 개사상자 & & & & $\mathrm{O}$ & & \\
\hline 292 & Centella asiatica (L.) Urb. 병풀 KP217374 & & & & & & $\mathrm{O}$ \\
\hline 293 & Cnidium japonicum Miq. 갯사상자 KP255725 & & & & $\mathrm{O}$ & & $\mathrm{O}$ \\
\hline 294 & Glehnia littoralis F. Schmidt ex Miq. 갯방풍 KP290414 & & & & & & $\mathrm{O}$ \\
\hline 295 & Hydrocotyle maritima Honda 선피막이 KP290387 & & & $\mathrm{O}$ & $\mathrm{O}$ & $\mathrm{O}$ & $\mathrm{O}$ \\
\hline 296 & Hydrocotyle ramiflora Maxim. 큰피막이 KP255665 & $\mathrm{O}$ & & & $\mathrm{O}$ & & $\mathrm{O}$ \\
\hline 297 & Hydrocotyle sibthorpioides Lam. 피막이 & & & & $\mathrm{O}$ & $\mathrm{O}$ & \\
\hline 298 & Oenanthe javanica (Blume) DC. 미나리 & & & & $\mathrm{O}$ & & \\
\hline 299 & Peucedanum terebinthaceum (Fisch. ex Trevir.) Fisch. ex Turcz. 기름나물 KP256171 & $\mathrm{O}$ & & & $\mathrm{O}$ & $\mathrm{O}$ & $\mathrm{O}$ \\
\hline 300 & Sium ninsi L. 감자개발나물 KP255773 & & & & $\mathrm{O}$ & $\mathrm{O}$ & $\mathrm{O}$ \\
\hline 301 & Sium suave Walter 개발나물 KP256172 & & & & $\mathrm{O}$ & & $\mathrm{O}$ \\
\hline \multirow[t]{2}{*}{302} & Torilis japonica (Houtt.) DC. 사상자 KP255359 & & & $\mathrm{O}$ & $\mathrm{O}$ & $\mathrm{O}$ & $\mathrm{O}$ \\
\hline & Loganiaceae 마전과 & & & & & & \\
\hline \multirow[t]{2}{*}{303} & Mitrasacme pygmaea R. Br. 큰벼룩아재비 KP319777 & & & & $\mathrm{O}$ & $\mathrm{O}$ & $\mathrm{O}$ \\
\hline & Gentianaceae 용담과 & & & & & & \\
\hline 304 & Gentiana squarrosa Ledeb. 구슬붕이 & & & & $\mathrm{O}$ & & \\
\hline \multirow[t]{2}{*}{305} & Gentiana zollingeri Fawc. 큰구슬붕이 KP255454 & & & & & & $\mathrm{O}$ \\
\hline & Apocynaceae 협죽도과 & & & & & & \\
\hline 306 & Apocynum lancifolium Russanov 개정향풀 & & & & & $\mathrm{O}$ & \\
\hline \multirow[t]{2}{*}{307} & Trachelospermum asiaticum (Siebold \& Zucc.) Nakai 마삭줄 & & & $\mathrm{O}$ & & $\mathrm{O}$ & \\
\hline & Asclepiadaceae 박주가리과 & & & & & & \\
\hline 308 & Cynanchum nipponicum Matsum. 덩굴박주가리 KP366308 & & & & & & $\mathrm{O}$ \\
\hline 309 & Cynanchum paniculatum (Bunge) Kitag. 산해박 KP290408 & $\mathrm{O}$ & & $\mathrm{O}$ & & $\mathrm{O}$ & $\mathrm{O}$ \\
\hline \multirow[t]{2}{*}{310} & Metaplexis japonica (Thunb.) Makino 박주가리 KP255708 & $\mathrm{O}$ & & & $\mathrm{O}$ & & $\mathrm{O}$ \\
\hline & Solanaceae 가지과 & & & & & & \\
\hline 311 & Datura tatula L. 독말풀 & & $\mathrm{O}$ & & & & \\
\hline
\end{tabular}


Appendix 1. Continued.

\begin{tabular}{|c|c|c|c|c|c|c|c|}
\hline \multirow{2}{*}{ No. } & \multirow{2}{*}{$\begin{array}{l}\text { Taxa and representative voucher specimen number } \\
\text { (KP: KOSPVP0000-, KN: KONPVP0000-, NP: NIBRVP0000-) }\end{array}$} & \multicolumn{6}{|c|}{ Study } \\
\hline & & 1 & 2 & 3 & 4 & 5 & 6 \\
\hline 312 & Lycium chinense Mill. 구기자나무 & & & & (C) & & \\
\hline & Physalis angulata L. 땅꽈리 & & & & $\mathrm{O}$ & & \\
\hline 314 & Solanum lyratum Thunb. 배풍등 & & & & $\mathrm{O}$ & & \\
\hline & Solanum nigrum L. 까마중 KP319804 & & & & & & $\mathrm{O}$ \\
\hline
\end{tabular}

\section{Convolvulaceae 메꽃과}

316 Calystegia hederacea Wall. 애기메꽃

$\mathrm{O}$

317 Calystegia sepium (L.) R. Br. var. japonica Makino 메꽃 KP255558

318 Calystegia soldanella (L.) Roem. \& Schult. 갯메꽃 KP255579

319 Cuscuta pentagona Engelm. 미국실새삼 KP255709

320 Ipomoea hederacea Jacq. 미국나팔꽃

$\mathrm{O}$

$\mathrm{O}$

321 Ipomoea hederacea Jacq. var. integriuscula A. Gray 둥근잎미국나팔꽃

$\mathrm{O}$

322 Ipomoea purpurea(L.) Roth 둥근잎나팔꽃 KP256205

$\mathrm{O}$

323 Pharbitis nil (L.) Choisy 나팔꽃 KP256272

$\mathrm{O}$

324 Quamoclit coccinea Moench 둥근잎유홍초

Polemoniaceae 꽃고비과

325 Phlox subulata L. 지면패랭이꽃 NP582551

Boraginaceae 지치과

326 Bothriospermum secundum Maxim. 참꽃받이

327 Bothriospermum tenellum (Hornem.) Fisch. \& C. A. Mey. 꽃받이 KP255351

328 Lithospermum zollingeri A. DC. 반디지치 KP255435

329 Symphytum officinale L. 컴프리

330 Trigonotis peduncularis (Trevis.) Benth. ex Baker \& S. Moore 꽃마리 KP318946

Verbenaceae 마편초과

331 Callicarpa japonica Thunb. 작살나무 KP255618

$\mathrm{O}$

$\mathrm{O}$

332 Callicarpa mollis Siebold \& Zucc. 새비나무 KP291299

O O

333 Caryopteris incana (Thunb. ex Houtt.) Miq. 층꽃나무 KP256134

$\mathrm{O}$

334 Verbena officinalis L. 마편초 KP234674

$\mathrm{O} \quad \mathrm{O}$

335 Vitex rotundifolia L. f. 순비기나무 KP256177

\section{Labiatae 꿀풀과}

336 Ajuga decumbens Thunb. 금창초 KP255451

337 Clinopodium chinense (Benth.) Kuntze 층층이꽃

$\mathrm{O}$

338 Clinopodium gracile (Benth.) Matsum. var. multicaule (Maxim.) Ohwi 탑꽃 KP255737

$\mathrm{O}$

339 Isodon inflexus (Thunb.) Kudô 산박하 KP255733

$\mathrm{O}$

O O O

340 Lamium amplexicaule L. 광대나물 KP255399

$\begin{array}{lll}\mathrm{O} & \mathrm{O} & \mathrm{O}\end{array}$

341 Leonurus japonicus Houtt. 익모초 KP256141

$\mathrm{O} \quad \mathrm{O}$

342 Lycopus lucidus Turcz. ex Benth. 쉽싸리

343 Lycopus maackianus (Maxim. ex Herder) Makino 애기쉽싸리

$\mathrm{O} \mathrm{O}$

344 Mentha arvensis L. var. piperascens Malinv. ex Holmes 박하 KP255753 
Appendix 1. Continued.

\begin{tabular}{|c|c|c|c|c|c|c|c|}
\hline \multirow{2}{*}{ No. } & \multirow{2}{*}{$\begin{array}{c}\text { Taxa and representative voucher specimen number } \\
\text { (KP: KOSPVP0000-, KN: KONPVP0000-, NP: NIBRVP0000-) }\end{array}$} & \multicolumn{6}{|c|}{ Study } \\
\hline & & 1 & 2 & 3 & 4 & 5 & 6 \\
\hline 346 & Mosla dianthera (Buch.-Ham. ex Roxb.) Maxim. 쥐깨풀 KP256174 & & & & & & $\mathrm{O}$ \\
\hline 347 & Mosla japonica (Benth. ex Oliv.) Maxim. 산들깨 & & & & & $\mathrm{O}$ & \\
\hline 348 & Mosla punctulata (J. F. Gmel.) Nakai 들깨풀 KP256240 & & & & & $\mathrm{O}$ & $\mathrm{O}$ \\
\hline 349 & Nepeta cataria L. 개박하 & $\mathrm{O}$ & & & & & \\
\hline 350 & Prunella asiatica Nakai 꿀풀 KP255527 & $\mathrm{O}$ & & $\mathrm{O}$ & $\mathrm{O}$ & $\mathrm{O}$ & $\mathrm{O}$ \\
\hline 351 & Salvia japonica Thunb. 둥근배암차즈기 & & & $\mathrm{O}$ & & $\mathrm{O}$ & \\
\hline 352 & Salvia plebeia R. Br. 배암차즈기 KP217363 & & & & $\mathrm{O}$ & & $\mathrm{O}$ \\
\hline 353 & Scutellaria fauriei H. Lév. \& Vaniot 그늘골무꽃 & & & & & $\mathrm{O}$ & \\
\hline 354 & Scutellaria indica L. 골무꽃 KP255428 & $\mathrm{O}$ & & $\mathrm{O}$ & $\mathrm{O}$ & $\mathrm{O}$ & $\mathrm{O}$ \\
\hline \multirow[t]{2}{*}{355} & Scutellaria strigillosa Hemsl. 참골무꽃 KP255517 & & & & & & $\mathrm{O}$ \\
\hline & Callitrichaceae 별이끼과 & & & & & & \\
\hline \multirow[t]{2}{*}{356} & Callitriche palustris L. 물별이끼 & & & & $\mathrm{O}$ & & \\
\hline & Plantaginaceae 질경이과 & & & & & & \\
\hline 357 & Plantago asiatica L. 질경이 & & & $\mathrm{O}$ & $\mathrm{O}$ & $\mathrm{O}$ & \\
\hline 358 & Plantago lanceolata L. 창질경이 KP255363 & $\mathrm{O}$ & $\mathrm{O}$ & & $\mathrm{O}$ & & $\mathrm{O}$ \\
\hline \multirow[t]{2}{*}{359} & Plantago major L. for. yezomariima (Koidz.) Ohwi 갯질경이 & & & & $\mathrm{O}$ & & \\
\hline & Oleaceae 물푸레나무과 & & & & & & \\
\hline 360 & Forsythia koreana (Rdhder) Nakai 개나리 NP582537 & (a) & & & & & (a) \\
\hline 361 & Fraxinus rhynchophylla Hance 물푸레나무 & & & & & $\mathrm{O}$ & \\
\hline 362 & Ligustrum japonicum Thunb. 광나무 KP255433 & & & & & & $\mathrm{O}$ \\
\hline \multirow[t]{2}{*}{363} & Ligustrum obtusifolium Siebold \& Zucc. 쥐똥나무 KP255460 & & & $\mathrm{O}$ & $\mathrm{O}$ & $\mathrm{O}$ & $\mathrm{O}$ \\
\hline & Scrophulariaceae 현삼과 & & & & & & \\
\hline 364 & Centranthera cochinchinensis (Lour.) Merr. var. lutea (H. Hara) H. Hara 성주풀 KP255771 & & & & & $\mathrm{O}$ & $\mathrm{O}$ \\
\hline 365 & Dopatrium junceum (Roxb.) Buch.-Ham. ex Benth. 등에풀 & & & & $\mathrm{O}$ & & \\
\hline 366 & Lindernia procumbens (Krock.) Borbás 밭뚝외풀 & & & & $\mathrm{O}$ & & \\
\hline 367 & Mazus pumilus (Burm. f.) Steenis 주름잎 KP255384 & & & & $\mathrm{O}$ & & $\mathrm{O}$ \\
\hline 368 & Melampyrum roseum Maxim. 꽃며느리밥풀 KP255774 & & & & $\mathrm{O}$ & $\mathrm{O}$ & $\mathrm{O}$ \\
\hline 369 & Paulownia coreana Uyeki 오동나무 KP255355 & & & & & & $\mathrm{O}$ \\
\hline 370 & Pseudolysimachion linariifolium (Pall. ex Link) Holub 꼬리풀 KP256132 & & & & & $\mathrm{O}$ & $\mathrm{O}$ \\
\hline 371 & Siphonostegia chinensis Benth. 절국대 KP234676 & & & & $\mathrm{O}$ & & $\mathrm{O}$ \\
\hline 372 & Veronica arvensis L. 선개불알풀 KP255352 & & & & $\mathrm{O}$ & & $\mathrm{O}$ \\
\hline 373 & Veronica persica Poir. 큰개불알풀 KP255339 & & $\mathrm{O}$ & & $\mathrm{O}$ & $\mathrm{O}$ & $\mathrm{O}$ \\
\hline \multirow[t]{2}{*}{374} & Veronica polita Fr. subsp. lilacina (H. Hara ex T. Yamaz.) T. Yamaz. 개불알풀 & & $\mathrm{O}$ & & & & \\
\hline & Acanthaceae 쥐꼬리망초과 & & & & & & \\
\hline \multirow[t]{2}{*}{375} & Justicia procumbens L. 쥐꼬리망초 KP256164 & & & & $\mathrm{O}$ & & $\mathrm{O}$ \\
\hline & Lentibulariaceae 통발과 & & & & & & \\
\hline 376 & Utricularia bifida L. 땅귀개 KP256122 & & & & $\mathrm{O}$ & $\mathrm{O}$ & $\mathrm{O}$ \\
\hline 377 & Utricularia japonica Makino 통발 & & & & $\mathrm{O}$ & & \\
\hline 378 & Utricularia pilosa (Makino) Makino 들통발 & & & & & $\mathrm{O}$ & \\
\hline
\end{tabular}


Appendix 1. Continued.

\begin{tabular}{|c|c|c|c|c|c|c|c|}
\hline \multirow{2}{*}{ No. } & \multirow{2}{*}{$\begin{array}{c}\text { Taxa and representative voucher specimen number } \\
\text { (KP: KOSPVP0000-, KN: KONPVP0000-, NP: NIBRVP0000-) }\end{array}$} & \multicolumn{6}{|c|}{ Study } \\
\hline & & 1 & 2 & 3 & 4 & 5 & 6 \\
\hline 379 & Utricularia racemosa Wall. ex Walp. 이삭귀개 KP255768 & & & & $\mathrm{O}$ & $\mathrm{O}$ & $\mathrm{O}$ \\
\hline \multirow[t]{2}{*}{380} & Utricularia tenuicaulis Miki 참통발 KP319796 & & & & & & $\mathrm{O}$ \\
\hline & Campanulaceae 초롱꽃과 & & & & & & \\
\hline 381 & Adenophora polyantha Nakai 수원잔대 & & & & & $\mathrm{O}$ & \\
\hline 382 & Adenophora remotiflora (Siebold \& Zucc.) Miq. 모시대 & & & & & $\mathrm{O}$ & \\
\hline 383 & Adenophora stricta Miq. 당잔대 KP320395 & & & & & & $\mathrm{O}$ \\
\hline 384 & Adenophora triphylla (Thunb.) A. DC. var. japonica (Regel) H. Hara 잔대 & $\mathrm{O}$ & & & $\mathrm{O}$ & & \\
\hline 385 & Adenophora verticillata Fisch. 층층잔대 KP255735 & & & & & $\mathrm{O}$ & $\mathrm{O}$ \\
\hline 386 & Lobelia chinensis Lour. 수염가래꽃 KP319794 & & & & $\mathrm{O}$ & & $\mathrm{O}$ \\
\hline \multirow[t]{2}{*}{387} & Platycodon grandiflorum (Jacq.) A. DC. 도라지 KP255700 & $\mathrm{O}$ & & & $\mathrm{O}$ & $\mathrm{O}$ & $\mathrm{O}$ \\
\hline & Rubiaceae 꼭두선이과 & & & & & & \\
\hline 388 & Asperula odorata L. 선갈퀴 & & & & $\mathrm{O}$ & & \\
\hline 389 & Galium pogonanthum Franch. \& Sav. 산갈퀴 KP255516 & & & & & & $\mathrm{O}$ \\
\hline 390 & Galium spurium L. var. echinospermum (Wallr.) Hayek 갈퀴덩굴 KP255343 & & & & $\mathrm{O}$ & & $\mathrm{O}$ \\
\hline 391 & Galium verum L. var. asiaticum Nakai 솔나물 KP255747 & $\mathrm{O}$ & & $\mathrm{O}$ & $\mathrm{O}$ & $\mathrm{O}$ & $\mathrm{O}$ \\
\hline 392 & Paederia scandens (Lour.) Merr. 계요등 KP234675 & $\mathrm{O}$ & & & $\mathrm{O}$ & $\mathrm{O}$ & $\mathrm{O}$ \\
\hline 393 & Paederia scandens (Lour.) Merr. var. angustifolia (Nakai) T. B. Lee 좁은잎계요등 & & & & $\mathrm{O}$ & & \\
\hline \multirow[t]{2}{*}{394} & Rubia cordifolia L. var. pratensis Maxim. 갈퀴꼭두선이 KP256143 & & & & $\mathrm{O}$ & & $\mathrm{O}$ \\
\hline & Diervillaceae 병꽃나무과 & & & & & & \\
\hline \multirow[t]{2}{*}{395} & Weigela subsessilis (Nakai) L. H. Bailey 병꽃나무 KP255446 & & & & & & $\mathrm{O}$ \\
\hline & Caprifoliaceae 인동과 & & & & & & \\
\hline \multirow[t]{2}{*}{396} & Lonicera japonica Thunb. 인동 KP255568 & $\mathrm{O}$ & & $\mathrm{O}$ & $\mathrm{O}$ & $\mathrm{O}$ & $\mathrm{O}$ \\
\hline & Viburnaceae 산분꽃나무과 & & & & & & \\
\hline 397 & Viburnum dilatatum Thunb. 가막살나무 & $\mathrm{O}$ & & & & & \\
\hline \multirow[t]{2}{*}{398} & Viburnum erosum Thunb. 덜뀡나무 KP255420 & & & $\mathrm{O}$ & & $\mathrm{O}$ & $\mathrm{O}$ \\
\hline & Valerianaceae 마타리과 & & & & & & \\
\hline 399 & Patrinia scabiosifolia Fisch. ex Trevir. 마타리 KP255702 & & & & $\mathrm{O}$ & $\mathrm{O}$ & $\mathrm{O}$ \\
\hline \multirow[t]{2}{*}{400} & Patrinia villosa (Thunb.) Juss. 뚝갈 KP256130 & $\mathrm{O}$ & & & $\mathrm{O}$ & $\mathrm{O}$ & $\mathrm{O}$ \\
\hline & Asteraceae 국화과 & & & & & & \\
\hline 401 & Ambrosia artemisiifolia L. 돼지풀 & $\mathrm{O}$ & $\mathrm{O}$ & & $\mathrm{O}$ & & \\
\hline 402 & Artemisia annua L. 개똥쑥 & & & & $\mathrm{O}$ & & \\
\hline 403 & Artemisia capillaris Thunb. 사철쑥 KP255727 & & & & $\mathrm{O}$ & & $\mathrm{O}$ \\
\hline 404 & Artemisia feddei H. Lèv. \& Vaniot 뺑쑥 & & & & $\mathrm{O}$ & & \\
\hline 405 & Artemisia japonica Thunb. 제비쑥 KP256131 & $\mathrm{O}$ & & $\mathrm{O}$ & $\mathrm{O}$ & $\mathrm{O}$ & $\mathrm{O}$ \\
\hline 406 & Artemisia keiskeana Miq. 맑은대쑥 KP291292 & $\mathrm{O}$ & & & $\mathrm{O}$ & $\mathrm{O}$ & $\mathrm{O}$ \\
\hline 407 & Artemisia princeps Pamp. 쑥 KP256093 & $\mathrm{O}$ & & & $\mathrm{O}$ & $\mathrm{O}$ & $\mathrm{O}$ \\
\hline 408 & Aster meyendorffii (Regel \& Maack) Voss 개쑥부쟁이 KP256142 & & & & & & $\mathrm{O}$ \\
\hline 409 & Aster hispidus Thunb. 갯쑥부쟁이 KP256089 & & & & $\mathrm{O}$ & & $\mathrm{O}$ \\
\hline 410 & Aster pilosus Willd. 미국쑥부쟁이 KP256187 & & & & & & $\mathrm{O}$ \\
\hline
\end{tabular}


Appendix 1. Continued.

\begin{tabular}{|c|c|c|c|c|c|c|c|}
\hline \multirow{2}{*}{ No. } & \multirow{2}{*}{$\begin{array}{l}\text { Taxa and representative voucher specimen number } \\
\text { (KP: KOSPVP0000-, KN: KONPVP0000-, NP: NIBRVP0000-) }\end{array}$} & \multicolumn{6}{|c|}{ Study } \\
\hline & & 1 & 2 & 3 & 4 & 5 & 6 \\
\hline & Aster scaber Thunb. 참취 KP256109 & $\mathrm{O}$ & & & $\mathrm{O}$ & $\mathrm{O}$ & $\mathrm{O}$ \\
\hline 412 & Aster subulatus Michx. 비짜루국화 KP256101 & & & & $\mathrm{O}$ & & $\mathrm{O}$ \\
\hline 413 & Aster subulatus Michx. var. sandwicensis A. G. Jones 큰비짜루국화 & & $\mathrm{O}$ & & $\mathrm{O}$ & & \\
\hline 414 & Aster tripolium L. 갯개미취 $\mathrm{KP} 256208$ & & & & & & $\mathrm{O}$ \\
\hline 415 & Aster yomena (Kitam.) Honda 쑥부쟁이 KP255754 & $\mathrm{O}$ & & & & & $\mathrm{O}$ \\
\hline 416 & Atractylodes ovata (Thunb.) DC. 삽주 KP256247 & $\mathrm{O}$ & & & & $\mathrm{O}$ & $\mathrm{O}$ \\
\hline 417 & Bidens bipinnata L. 도깨비바늘 KP256178 & & & & $\mathrm{O}$ & & $\mathrm{O}$ \\
\hline 418 & Bidens frondosa L. 미국가막사리 & & & & $\mathrm{O}$ & $\mathrm{O}$ & \\
\hline 419 & Bidens pilosa L. 울산도깨비바늘 KP256140 & & & & & & $\mathrm{O}$ \\
\hline 420 & Breea segeta (Bunge) Kitam. 조뱅이 KP255596 & & & & $\mathrm{O}$ & & $\mathrm{O}$ \\
\hline 421 & Carduus crispus L. 지느러미엉겅퀴 & & & & $\mathrm{O}$ & & \\
\hline 422 & Carpesium abrotanoides L. 담배풀 & & & & & $\mathrm{O}$ & \\
\hline 423 & Carpesium cernuum L. 좀담배풀 & & & & $\mathrm{O}$ & $\mathrm{O}$ & \\
\hline 424 & Carpesium divaricatum Siebold \& Zucc. 긴담배풀 KP255748 & & & & & & $\mathrm{O}$ \\
\hline 425 & Centipeda minima (L.) A. Braun \& Asch. 중대가리풀 KP319783 & & & & $\mathrm{O}$ & & $\mathrm{O}$ \\
\hline 426 & Chrysanthemum leucanthemum L. 불란서국화 & & & & $\mathrm{O}$ & & \\
\hline 427 & Cirsium japonicum (Thunb.) Fisch. ex DC. 엉겅퀴 KP255634 & $\mathrm{O}$ & & & $\mathrm{O}$ & & $\mathrm{O}$ \\
\hline 428 & Conyza bonariensis (L.) Cronquist 실망초 KP255724 & & $\mathrm{O}$ & & & & $\mathrm{O}$ \\
\hline 429 & Conyza canadensis (L.) Cronquist 망초 KP255719 & & $\mathrm{O}$ & & & & $\mathrm{O}$ \\
\hline 430 & Conyza sumatrensis (Retz.) E. Walker 큰망초 & & $\mathrm{O}$ & & $\mathrm{O}$ & & \\
\hline 431 & Coreopsis lanceolata L. 큰금계국 KP255508 & $\mathrm{O}$ & $\mathrm{O}$ & & $\mathrm{O}$ & & $\mathrm{O}$ \\
\hline 432 & Coreopsis tinctoria Nutt. 기생초 & & $\mathrm{O}$ & & & & \\
\hline 433 & Cosmos bipinnatus Cav. 코스모스 & $\mathrm{O}$ & $\mathrm{O}$ & & & & \\
\hline & Cosmos sulphureus Cav. 노랑코스모스 & & $\mathrm{O}$ & & & & \\
\hline 435 & Crassocephalum crepidioides (Benth.) S. Moore 주홍서나물 KP256148 & $\mathrm{O}$ & & & & & $\mathrm{O}$ \\
\hline 436 & Dendranthema boreale (Makino) Ling ex Kitam. 산국 KP291302 & & & & & & $\mathrm{O}$ \\
\hline 437 & Dendranthema indicum (L.) Des Moul. 감국 & $\mathrm{O}$ & & & & & \\
\hline 438 & Dendranthema zawadskii (Herbich) Tzvelev var. latilobum (Maxim.) Kitam. 구절초 & $\mathrm{O}$ & & & & & \\
\hline 439 & Eclipta prostrata (L.) L. 한련초 KP255671 & & & & $\mathrm{O}$ & & $\mathrm{O}$ \\
\hline 440 & Erechtites hieraciifolia (L.) Raf. ex DC. 붉은서나물 KP256181 & & $\mathrm{O}$ & & & & $\mathrm{O}$ \\
\hline & Erigeron annuus (L.) Pers. 개망초 KP255629 & $\mathrm{O}$ & $\mathrm{O}$ & $\mathrm{O}$ & $\mathrm{O}$ & $\mathrm{O}$ & $\mathrm{O}$ \\
\hline 442 & Euchiton japonicus (Thunb.) Holub 풀솜나물 KP255385 & $\mathrm{O}$ & & $\mathrm{O}$ & $\mathrm{O}$ & $\mathrm{O}$ & $\mathrm{O}$ \\
\hline & Eupatorium japonicum Thunb. 등골나물 & $\mathrm{O}$ & & & & & \\
\hline 444 & Eupatorium lindleyanum DC. 골등골나물 KP319826 & & & & $\mathrm{O}$ & $\mathrm{O}$ & $\mathrm{O}$ \\
\hline & $\begin{array}{l}\text { Eupatorium makinoi T. Kawahara \& Yahara var. oppositifolium T. Kawahara \& Yahara 벌등골나물 } \\
\text { KP255721 }\end{array}$ & $\mathrm{O}$ & & & $\mathrm{O}$ & & $\mathrm{O}$ \\
\hline 446 & Eupatorium tripartitum (Makino) Murata \& H. Koyama 향등골나물 KP319755 & & & & $\mathrm{O}$ & & $\mathrm{O}$ \\
\hline 447 & Galinsoga parviflora Cav. 별꽃아재비 & & $\mathrm{O}$ & & & & \\
\hline 448 & Gamochaeta calviceps (Fernald) Cabrera 선풀솜나물 & & & & $\mathrm{O}$ & & \\
\hline
\end{tabular}


Appendix 1. Continued.

\begin{tabular}{|c|c|c|c|c|c|c|c|}
\hline \multirow{2}{*}{ No. } & \multirow{2}{*}{$\begin{array}{c}\text { Taxa and representative voucher specimen number } \\
\text { (KP: KOSPVP0000-, KN: KONPVP0000-, NP: NIBRVP0000-) }\end{array}$} & \multicolumn{6}{|c|}{ Study } \\
\hline & & 1 & 2 & 3 & 4 & 5 & 6 \\
\hline & Helianthus tuberosus L. 뚱딴지 & & $\mathrm{O}$ & & & & \\
\hline 450 & Hemistepta lyrata Bunge 지칭개 KP255608 & & & & $\mathrm{O}$ & & $\mathrm{O}$ \\
\hline 451 & Hieracium umbellatum L. 조밥나물 KP255693 & & & & & & $\mathrm{O}$ \\
\hline 452 & Hololeion maximowiczii Kitam. 께묵 KP256176 & & & & & $\mathrm{O}$ & $\mathrm{O}$ \\
\hline 453 & Hypochaeris radicata L. 서양금혼초 $\mathrm{KP} 255729$ & & & & $\mathrm{O}$ & & $\mathrm{O}$ \\
\hline 454 & Ixeridium dentatum (Thunb.) Tzvelev 씀바귀 KP255444 & & & $\mathrm{O}$ & $\mathrm{O}$ & $\mathrm{O}$ & $\mathrm{O}$ \\
\hline 455 & Ixeris chinensis (Thunb.) Kitag. subsp. strigosa (H. Lèv. \& Vaniot) Kitam. 선씀바귀 & & & & $\mathrm{O}$ & & \\
\hline 456 & Ixeris debilis (Thunb.) A. Gray 벋음씀바귀 KP255371 & & & & $\mathrm{O}$ & & $\mathrm{O}$ \\
\hline 457 & Lactuca indica L. var. laciniata H. Hara 왕고들빼기 KP255691 & & & & $\mathrm{O}$ & & $\mathrm{O}$ \\
\hline 458 & Lactuca scariola L. 가시상추 & & $\mathrm{O}$ & & $\mathrm{O}$ & & \\
\hline 459 & Petasites japonicus (Siebold \& Zucc.) Maxim. 머위 NP582546 & & & & & $\mathrm{O}$ & $\mathrm{O}$ \\
\hline 460 & Pseudognaphalium affine (D. Don) Anderb. 떡쑥 KP255574 & & & $\mathrm{O}$ & $\mathrm{O}$ & $\mathrm{O}$ & $\mathrm{O}$ \\
\hline 461 & Pseudognaphalium hypoleucum (DC.) Hilliard \& B. L. Burtt 금떡쑥 & & & & $\mathrm{O}$ & & \\
\hline 462 & Rudbeckia hirta L. var. pulcherrima Farw. 원추천인국 & & $\mathrm{O}$ & & & & \\
\hline 463 & Saussurea japonica (Thunb.) DC. 큰각시취 & & & & $\mathrm{O}$ & & \\
\hline 464 & Saussurea pulchella (Fisch.) Fisch. ex Colla 각시취 & & & & & $\mathrm{O}$ & \\
\hline 465 & Saussurea seoulensis Nakai 분취 & $\mathrm{O}$ & & & & & \\
\hline 466 & Senecio vulgaris L. 개쑥갓 KP255552 & & $\mathrm{O}$ & $\mathrm{O}$ & $\mathrm{O}$ & $\mathrm{O}$ & $\mathrm{O}$ \\
\hline 467 & Serratula coronata L. subsp. insularis (Iljin) Kitam. 산비장이 KP256255 & & & & & $\mathrm{O}$ & $\mathrm{O}$ \\
\hline 468 & Sigesbeckia glabrescens Makino 진득찰 KP255672 & & & & & & $\mathrm{O}$ \\
\hline 469 & Sigesbeckia pubescens (Makino) Makino 털진득찰 KP256227 & & & & $\mathrm{O}$ & & $\mathrm{O}$ \\
\hline 470 & Solidago altissima L. 양미역취 KP256207 & & $\mathrm{O}$ & & & & $\mathrm{O}$ \\
\hline 471 & Solidago gigantea Aiton subsp. serotina (Aiton) McNeill 미국미역취 KP255681 & & & & & & $\mathrm{O}$ \\
\hline 472 & Solidago virgaurea L. subsp. asiatica Kitam. ex Hara 미역취 KP291290 & $\mathrm{O}$ & & & & $\mathrm{O}$ & $\mathrm{O}$ \\
\hline 473 & Sonchus asper (L.) Hill 큰방가지똥 KP318911 & & $\mathrm{O}$ & & $\mathrm{O}$ & & $\mathrm{O}$ \\
\hline 474 & Sonchus brachyotus DC. 사데풀 KP256102 & & & & $\mathrm{O}$ & & $\mathrm{O}$ \\
\hline 475 & Sonchus oleraceus L. 방가지똥 KP255407 & & $\mathrm{O}$ & $\mathrm{O}$ & $\mathrm{O}$ & $\mathrm{O}$ & $\mathrm{O}$ \\
\hline 476 & Syneilesis palmata (Thunb.) Maxim. 우산나물 & $\mathrm{O}$ & & & & & \\
\hline 477 & Tagetes minuta L. 만수국아재비 & & $\mathrm{O}$ & & & & \\
\hline 478 & Taraxacum laevigatum (Willd.) DC. 붉은씨서양민들레 & & $\mathrm{O}$ & & & & \\
\hline 479 & Taraxacum officinale F. H. Wigg. 서양민들레 KP255354 & & $\mathrm{O}$ & & & & $\mathrm{O}$ \\
\hline 480 & Taraxacum platycarpum Dahlst. 민들레 KP255545 & & & & $\mathrm{O}$ & & $\mathrm{O}$ \\
\hline 481 & Tephroseris kirilowii (Turcz. ex DC.) Holub 솜방망이 KP255443 & & & & & $\mathrm{O}$ & $\mathrm{O}$ \\
\hline & Tephroseris pseudosonchus (Vaniot) C. Jeffrey \& Y. L. Chen 물솜방망이 & & & & $\mathrm{O}$ & & \\
\hline 483 & Xanthium canadense Mill. 큰도고마리 & & $\mathrm{O}$ & & & & \\
\hline 484 & Xanthium strumarium L. 도꼬마리 & & $\mathrm{O}$ & & & & \\
\hline 485 & Youngia japonica (L.) DC. 뽀리뱅이 KP255353 & & & $\mathrm{O}$ & $\mathrm{O}$ & $\mathrm{O}$ & $\mathrm{O}$ \\
\hline & Alismataceae 택사과 & & & & & & \\
\hline & Alisma canaliculatum A. Braun \& Bouchè 택사 KP290402 & & & & & & $\mathrm{O}$ \\
\hline
\end{tabular}


Appendix 1. Continued.

\begin{tabular}{lllllll}
\hline \hline \multirow{2}{*}{ No. } & Taxa and representative voucher specimen number & \multicolumn{3}{c}{ Study } \\
\cline { 3 - 7 } & (KP: KOSPVP000-, KN: KONPVP0000-, NP: NIBRVP0000-) & 1 & 2 & 3 & 4 & 6
\end{tabular}

\section{Hydrocharitaceae 자라풀과}

487 Hydrilla verticillata (L. f.) Royle 검정말

488 Ottelia alismoides (L.) Pers. 물질경이 KP319840

$\mathrm{O}$

$\mathrm{O}$

Juncaginaceae 지채과

489 Triglochin maritimum L. 지채 KP255587

Potamogetonaceae 가래과

490 Potamogeton crispus L. 말즘 KP255593

491 Potamogeton cristatus Regel \& Maack 가는가래 KP319795

492 Potamogeton distinctus A. Benn. 가래 KP366313

493 Potamogeton pusillus L. 실말

\section{Ruppiaceae 줄말과}

494 Ruppia maritima L. 줄말

Najadaceae 나자스말과

495 Najas graminea Delile 나자스말

$\mathrm{O}$

496 Najas marina L. 민나자스말

\section{Araceae 천남성과}

497 Pinellia ternata (Thunb.) Breitenb. 반하

Lemnaceae 개구리밥과

498 Lemna perpusilla Torr. 좀개구리밥 NP582540

\section{Commelinaceae 닭의장풀과}

499 Commelina communis L. 닭의장풀 KP255647

500 Murdannia keisak (Hassk.) Hand.-Mazz. 사마귀풀 KN373330

501 Tradescantia ohiensis Raf. 자주달개비

Eriocaulaceae 곡정초과

502 Eriocaulon hondoense Satake 큰개수염 KP256168

503 Eriocaulon miquelianum Körn. 개수염 KP256261

\section{Juncaceae 골풀과}

504 Juncus diastrophanthus Buchenau 별날개골풀 KP256260

505 Juncus effusus L. var. decipiens Buchenau 골풀 KP256286

506 Juncus gracillimus (Buchenau) V. I. Krecz. \& Gontsch. 물골풀

507 Juncus krameri Franch. \& Sav. 비녀골풀

508 Juncus leschenaultii Gay ex Laharpe 참비녀골풀 KP256311

509 Juncus setchuensis Buchenau var. effusoides Buchenau 푸른갯골풀 KP256312

510 Juncus wallichianus Laharpe 눈비녀골풀 KP290400

511 Luzula capitata (Miq.) Miq. 뀡의밥 NP582531

\section{Cyperaceae 사초과}

512 Bolboschoenus maritimus (L.) Palla 매자기

$\mathrm{O}$

513 Bolboschoenus planiculmis (F.Schmidt) T. V. Egorova 새섬매자기 KP217357 
Appendix 1. Continued.

\begin{tabular}{|c|c|c|c|c|c|c|c|}
\hline \multirow{2}{*}{ No. } & \multirow{2}{*}{$\begin{array}{c}\text { Taxa and representative voucher specimen number } \\
\text { (KP: KOSPVP0000-, KN: KONPVP0000-, NP: NIBRVP0000-) }\end{array}$} & \multicolumn{6}{|c|}{ Study } \\
\hline & & 1 & 2 & 3 & 4 & 5 & 6 \\
\hline 515 & Carex appendiculata (Trautv. \& C. A. Mey.) Kük. 뚝사초 KP256307 & & & & & & $\mathrm{O}$ \\
\hline 516 & Carex breviculmis $\mathrm{R}$. Br. 청사초 & & & & $\mathrm{O}$ & & \\
\hline 517 & Carex brownii Tuck. 흰꼬리사초 KP256300 & & & & & & $\mathrm{O}$ \\
\hline 518 & Carex ciliatomarginata Nakai 털대사초 & & & & & $\mathrm{O}$ & \\
\hline 519 & Carex conica Boott 애기사초 & & & & $\mathrm{O}$ & & \\
\hline 520 & Carex fernaldiana H. Lèv. \& Vaniot 실사초 & $\mathrm{O}$ & & & & & \\
\hline 521 & Carex humilis Leyss. 산거울 & $\mathrm{O}$ & & & & & \\
\hline 522 & Carex kobomugi Ohwi 통보리사초 KP290415 & & & & & & $\mathrm{O}$ \\
\hline 523 & Carex lanceolata Boott 그늘사초 KP256299 & $\mathrm{O}$ & & & $\mathrm{O}$ & $\mathrm{O}$ & $\mathrm{O}$ \\
\hline 524 & Carex laticeps C. B. Clarke 갯보리사초 & & & & & $\mathrm{O}$ & \\
\hline 525 & Carex leucochlora Bunge 이삼사초 & & & & & $\mathrm{O}$ & \\
\hline 526 & Carex maculata Boott 무늬사초 & & & & & $\mathrm{O}$ & \\
\hline 527 & Carex maximowiczii Miq. 왕비늘사초 KP256285 & & & $\mathrm{O}$ & $\mathrm{O}$ & $\mathrm{O}$ & $\mathrm{O}$ \\
\hline 528 & Carex mitrata var. aristata Ohwi 겨락겨사초 & & & & & $\mathrm{O}$ & \\
\hline 529 & Carex nervata Franch. \& Sav. 양지사초 & & & & $\mathrm{O}$ & & \\
\hline 530 & Carex phacota Spreng. 비늘사초 & & & & $\mathrm{O}$ & & \\
\hline 531 & Carex polyschoena H. Lèv. \& Vaniot 가지청사초 & & & & $\mathrm{O}$ & $\mathrm{O}$ & \\
\hline 532 & Carex sabynensis Less. ex Kunth 실청사초 & & & & & $\mathrm{O}$ & \\
\hline 533 & Carex scabrifolia Steud. 천일사초 KP217356 & & & & $\mathrm{O}$ & & $\mathrm{O}$ \\
\hline 534 & Carex siderosticta Hance 대사초 KP255448 & & & & & & $\mathrm{O}$ \\
\hline 535 & Carex tristachya Thunb. 반들사초 & & & & $\mathrm{O}$ & $\mathrm{O}$ & \\
\hline 536 & Carex tristachya Thunb. var. pocilliformis (Boott) Kük. 애기반들사초 & & & $\mathrm{O}$ & & $\mathrm{O}$ & \\
\hline 537 & Cladium chinense Nees 층층고랭이 KP319550 & & & & $\mathrm{O}$ & $\mathrm{O}$ & $\mathrm{O}$ \\
\hline 538 & Cyperus difformis L. 알방동산이 KP256330 & & & & $\mathrm{O}$ & $\mathrm{O}$ & $\mathrm{O}$ \\
\hline 539 & Cyperus globosus Forssk. 드렁방동산이 & & & & $\mathrm{O}$ & $\mathrm{O}$ & \\
\hline 540 & Cyperus haspan L. 모기방동산이 KP319750 & & & & $\mathrm{O}$ & $\mathrm{O}$ & $\mathrm{O}$ \\
\hline 541 & Cyperus iria L. 참방동산이 $\mathrm{KP} 256228$ & & & & $\mathrm{O}$ & $\mathrm{O}$ & $\mathrm{O}$ \\
\hline 542 & Cyperus microiria Steud. 금방동산이 KP256219 & & & & $\mathrm{O}$ & $\mathrm{O}$ & $\mathrm{O}$ \\
\hline 543 & Cyperus nipponicus Franch. \& Sav. 푸른방동산이 & & & & $\mathrm{O}$ & & \\
\hline 544 & Cyperus sanguinolentus Vahl 방동산이대가리 KP366310 & $\mathrm{O}$ & & & & & $\mathrm{O}$ \\
\hline 545 & Cyperus tenuispica Steud. 우산방동산이 KP256332 & & & & & $\mathrm{O}$ & $\mathrm{O}$ \\
\hline 546 & Eleocharis kamtschatica (C. A. Mey.) Kom. 올방개아재비 KP256296 & & & & $\mathrm{O}$ & & $\mathrm{O}$ \\
\hline 547 & Eleocharis tetraquetra Nees 네모골 & & & & & $\mathrm{O}$ & \\
\hline 548 & Eleocharis wichurae Boeck. 좀네모골 KP319543 & & & & $\mathrm{O}$ & $\mathrm{O}$ & $\mathrm{O}$ \\
\hline 549 & Fimbristylis autumnalis (L.) Roem. \& Schult. 애기하늘지기 & & & & $\mathrm{O}$ & $\mathrm{O}$ & \\
\hline 550 & $\begin{array}{l}\text { Fimbristylis complanata (Retz.) Link var. exaltata (T. Koyama) Y. C. Tang ex S. R. Zhang \& T. } \\
\text { Koyama 어른지기 KP256322 }\end{array}$ & & & & & & $\mathrm{O}$ \\
\hline 551 & Fimbristylis cymosa R. Br. 바다지기 & & & & $\mathrm{O}$ & & \\
\hline 552 & Fimbristylis dichotoma (L.) Vahl 하늘지기 KP366601 & & & & $\mathrm{O}$ & $\mathrm{O}$ & $\mathrm{O}$ \\
\hline
\end{tabular}


Appendix 1. Continued.

\begin{tabular}{|c|c|c|c|c|c|c|c|}
\hline \multirow{2}{*}{ No. } & \multirow{2}{*}{$\begin{array}{c}\text { Taxa and representative voucher specimen number } \\
\text { (KP: KOSPVP0000-, KN: KONPVP0000-, NP: NIBRVP0000-) }\end{array}$} & \multicolumn{6}{|c|}{ Study } \\
\hline & & 1 & 2 & 3 & 4 & 5 & 6 \\
\hline 553 & Fimbristylis littoralis Gaudich. 바람하늘지기 & & & & $\mathrm{O}$ & $\mathrm{O}$ & \\
\hline 554 & Fimbristylis longispica Steud. 큰하늘지기 KP256313 & & & & $\mathrm{O}$ & & $\mathrm{O}$ \\
\hline 555 & Fimbristylis pierotii Miq. 들하늘지기 & & & $\mathrm{O}$ & & $\mathrm{O}$ & \\
\hline 556 & Fimbristylis sieboldii Miq. Ex Franch. \& Sav. 갯하늘지기 KP256315 & & & & $\mathrm{O}$ & $\mathrm{O}$ & $\mathrm{O}$ \\
\hline 557 & Fimbristylis squarrosa Vahl var. esquarrosa Makino 암하늘지기 & & & & & $\mathrm{O}$ & \\
\hline 558 & Fimbristylis tomentosa Vahl 털하늘지기 & & & & $\mathrm{O}$ & & \\
\hline 559 & Fimbristylis tristachya R. Br. var. subbispicata (Nees \& Meyen) T. Koyama 꼴하늘지기 KP256325 & & & & $\mathrm{O}$ & $\mathrm{O}$ & $\mathrm{O}$ \\
\hline 560 & Kyllinga brevifolia Rottb. 파대가리 KP256331 & & & & & $\mathrm{O}$ & $\mathrm{O}$ \\
\hline 561 & Rhynchospora chinensis Nees \& Meyen 고양이수염 KP256167 & & & & $\mathrm{O}$ & $\mathrm{O}$ & $\mathrm{O}$ \\
\hline 562 & Rhynchospora faberi C. B. Clarke 골풀아재비 KP256256 & & & & $\mathrm{O}$ & $\mathrm{O}$ & $\mathrm{O}$ \\
\hline 563 & Rhynchospora fauriei Franch. 큰고양이수염 & & & & & $\mathrm{O}$ & \\
\hline 564 & Rhynchospora fujiiana Makino 좀고양이수염 KP290390 & & & & $\mathrm{O}$ & & $\mathrm{O}$ \\
\hline 565 & Rhynchospora rubra (Lour.) Makino 붉은골풀아재비 KP319536 & & & & & & $\mathrm{O}$ \\
\hline 566 & Schoenoplectiella hotarui (Ohwi) J. Jung \& H.-K. Choi 좀올챙이골 KP319757 & & & & $\mathrm{O}$ & & $\mathrm{O}$ \\
\hline 567 & Schoenoplectiella juncoides (Roxb.) Lye 올챙이고랭이 & & & & $\mathrm{O}$ & $\mathrm{O}$ & \\
\hline 568 & Scirpus mitsukurianus Makino 솔방울골 & & & & & $\mathrm{O}$ & \\
\hline 569 & Scirpus wichurai Boeck. var. asiaticus (Beetle) T. Koyama ex Ohwi 방울고랭이 KP255772 & & & & & & $\mathrm{O}$ \\
\hline 570 & Scleria caricina (R. Br.) Benth. 애기개올미 KP366305 & & & & $\mathrm{O}$ & $\mathrm{O}$ & $\mathrm{O}$ \\
\hline 571 & Scleria parvula Steud. 너도고랭이 KP256268 & & & & $\mathrm{O}$ & $\mathrm{O}$ & $\mathrm{O}$ \\
\hline 572 & Scleria rugosa R. Br. 가시개올미 KP366306 & & & & $\mathrm{O}$ & $\mathrm{O}$ & $\mathrm{O}$ \\
\hline & Poaceae 화본과 & & & & & & \\
\hline 573 & Agropyron ciliare (Trin.) Franch. 속털개밀 KP318909 & & & $\mathrm{O}$ & $\mathrm{O}$ & $\mathrm{O}$ & $\mathrm{O}$ \\
\hline 574 & Agropyron tsukushiense (Honda) Ohwi var. transiens (Hack.) Ohwi 개밀 KP256306 & & & & $\mathrm{O}$ & & $\mathrm{O}$ \\
\hline 575 & Agrostis clavata Trin. var. nukabo Ohwi 겨이삭 & & & $\mathrm{O}$ & $\mathrm{O}$ & $\mathrm{O}$ & \\
\hline 576 & Alopecurus aepualis Sobol. 둑새풀 KP255345 & & & & & & $\mathrm{O}$ \\
\hline 577 & Alopecurus japonicus Steud. 털뚝새풀 KP256333 & & & & & & $\mathrm{O}$ \\
\hline 578 & Arthraxon hispidus (Thunb.) Makino 조개풀 KP256251 & & & & $\mathrm{O}$ & & $\mathrm{O}$ \\
\hline 579 & Arundinella hirta (Thunb.) Tanaka 새 KP256237 & $\mathrm{O}$ & & & $\mathrm{O}$ & $\mathrm{O}$ & $\mathrm{O}$ \\
\hline 580 & Arundinella hirta (Thunb.) Tanaka var. ciliata (Thunb.) Koidz. 털새 KP319773 & & & & $\mathrm{O}$ & $\mathrm{O}$ & $\mathrm{O}$ \\
\hline 581 & Arundinella hirta (Thunb.) Tanaka var. hondana Koidz. 이삭털새 & & & & $\mathrm{O}$ & & \\
\hline 582 & Avena fatua L. 메귀리 NP582544 & & $\mathrm{O}$ & & $\mathrm{O}$ & & $\mathrm{O}$ \\
\hline 583 & Beckmannia syzigachne (Steud.) Fernald 개피 KP255640 & & & & $\mathrm{O}$ & & $\mathrm{O}$ \\
\hline 584 & Briza minor L. 방울새풀 KP255518 & & & & & & $\mathrm{O}$ \\
\hline 585 & Bromus japonicus Thunb. 참새귀리 KP318928 & & & & $\mathrm{O}$ & & $\mathrm{O}$ \\
\hline 586 & Bromus pauciflorus (Thunb.) Hack. 꼬리새 KP256301 & & & & & & $\mathrm{O}$ \\
\hline 587 & Bromus rigidus Roth 긴까락빕새귀리 KP256294 & & & & & & $\mathrm{O}$ \\
\hline 588 & Bromus unioloides Kunth 큰이삭풀 KP318908 & & $\mathrm{O}$ & & $\mathrm{O}$ & & $\mathrm{O}$ \\
\hline 589 & Calamagrostis arundinacea (L.) Roth 실새풀 & & & & & $\mathrm{O}$ & \\
\hline 590 & Calamagrostis epigeios (L.) Roth 산조풀 KP290417 & & & & & & $\mathrm{O}$ \\
\hline
\end{tabular}


Appendix 1. Continued.

\begin{tabular}{|c|c|c|c|c|c|c|c|}
\hline \multirow{2}{*}{ No. } & \multirow{2}{*}{$\begin{array}{c}\text { Taxa and representative voucher specimen number } \\
\text { (KP: KOSPVP0000-, KN: KONPVP0000-, NP: NIBRVP0000-) }\end{array}$} & \multicolumn{6}{|c|}{ Study } \\
\hline & & 1 & 2 & 3 & 4 & 5 & 6 \\
\hline 591 & Capillipedium assimile (Steud.) A. Camus 나도기름새 KP256242 & & & & & $\mathrm{O}$ & $\mathrm{O}$ \\
\hline 592 & Cymbopogon tortilis (J. Presl) A. Camus subsp. goeringii (Steud.) T. Koyama 개솔새 KP256104 & & & & $\mathrm{O}$ & $\mathrm{O}$ & $\mathrm{O}$ \\
\hline 593 & Dactylis glomerata L. 오리새 KP256289 & & $\mathrm{O}$ & & $\mathrm{O}$ & & $\mathrm{O}$ \\
\hline 594 & Digitaria sanguinalis (L.) Scop. 바랭이 KP255661 & & & & $\mathrm{O}$ & $\mathrm{O}$ & $\mathrm{O}$ \\
\hline 595 & Dimeria ornithopoda Trin. 잔디바랭이 & & & & & $\mathrm{O}$ & \\
\hline 596 & Echinochloa crus-galli (L.) P. Beauv. 돌피 KP255628 & & & & $\mathrm{O}$ & & $\mathrm{O}$ \\
\hline 597 & Echinochloa crus-galli (L.) P. Beauv. var. echinata (Willd.) Honda 물피 KP319837 & & & & & & $\mathrm{O}$ \\
\hline 598 & Echinochloa crus-galli (L.) P. Beauv. var. praticola Ohwi 좀돌피 & & & & & $\mathrm{O}$ & \\
\hline 599 & Echinochloa oryzoides (Ard.) Fritsch 논피 & & & & & $\mathrm{O}$ & \\
\hline 600 & Eleusine indica (L.) Gaertn. 왕바랭이 KP255660 & & & & & & $\mathrm{O}$ \\
\hline 601 & Elymus dahuricus Turcz. ex Griseb. 갯보리 & & & $\mathrm{O}$ & & $\mathrm{O}$ & \\
\hline 602 & Eragrostis curvula (Schrad.) Nees 능수참새그령 & & $\mathrm{O}$ & & & & \\
\hline 603 & Eragrostis pilosa (L.) P. Beauv. 큰비노리 & & & & $\mathrm{O}$ & & \\
\hline 604 & Eulalia speciosa (Debeaux) Kuntze 개억새 KP320397 & & & & & $\mathrm{O}$ & $\mathrm{O}$ \\
\hline 605 & Festuca arundinacea Schreb. 큰김의털 & & $\mathrm{O}$ & & $\mathrm{O}$ & & \\
\hline 606 & Festuca myuros L. 들묵새 & & $\mathrm{O}$ & & $\mathrm{O}$ & & \\
\hline 607 & Festuca ovina L. 김의털 KP256231 & & & $\mathrm{O}$ & $\mathrm{O}$ & $\mathrm{O}$ & $\mathrm{O}$ \\
\hline 608 & Festuca parvigluma Steud. 김의털아재비 & & & & $\mathrm{O}$ & & \\
\hline 609 & Festuca rubra L. 왕김의털 & & & & $\mathrm{O}$ & & \\
\hline 610 & Hemarthria compressa (L. f.) R. Br. var. japonica (Hack.) Ohwi 쇠치기풀 & & & & $\mathrm{O}$ & & \\
\hline 611 & Hierochloe odorata (L.) P. Beauv. 향모 & & & & $\mathrm{O}$ & & \\
\hline 612 & Hordeum vulgare L. var. hexastichon (L.) Asch. 보리 & & & & (a) & & \\
\hline 613 & Imperata cylindrica (L.) Raeusch. var. koenigii (Retz.) Benth. ex Pilg. 띠 KP256282 & $\mathrm{O}$ & & $\mathrm{O}$ & $\mathrm{O}$ & $\mathrm{O}$ & $\mathrm{O}$ \\
\hline 614 & Isachne globosa (Thunb.) Kuntze 기장대풀 KP255638 & & & & $\mathrm{O}$ & $\mathrm{O}$ & $\mathrm{O}$ \\
\hline 615 & Ischaemum anthephoroides (Steud.) Miq. 갯쇠보리 KP290413 & & & & & & $\mathrm{O}$ \\
\hline 616 & Ischaemum crassipes (Steud.) Thell. 쇠보리 KP255646 & $\mathrm{O}$ & & & $\mathrm{O}$ & $\mathrm{O}$ & $\mathrm{O}$ \\
\hline 617 & Koeleria cristata Pers. 도랑이피 & & & $\mathrm{O}$ & $\mathrm{O}$ & $\mathrm{O}$ & \\
\hline 618 & Leersia japonica (Makino \& Honda) Honda 나도겨풀 & & & & $\mathrm{O}$ & & \\
\hline 619 & Leptochloa malabarica (L.) Veldkamp 갯드렁새 & & $\mathrm{O}$ & & $\mathrm{O}$ & & \\
\hline 620 & Lolium multiflorum Lam. 쥐보리 KP318929 & & $\mathrm{O}$ & $\mathrm{O}$ & $\mathrm{O}$ & $\mathrm{O}$ & $\mathrm{O}$ \\
\hline 621 & Lolium perenne L. 호밀풀 & & $\mathrm{O}$ & & $\mathrm{O}$ & & \\
\hline 622 & Melica nutans L. 왕쌀새 & & & & & $\mathrm{O}$ & \\
\hline 623 & Miscanthus sacchariflorus (Maxim.) Hack. 물억새 & $\mathrm{O}$ & & & & & \\
\hline 624 & Miscanthus sinensis Andersson 참억새 KP256097 & $\mathrm{O}$ & & & $\mathrm{O}$ & $\mathrm{O}$ & $\mathrm{O}$ \\
\hline 625 & Miscanthus sinensis Andersson for. gracillimus (Hitchc.) Ohwi 가는잎억새 & & & & $\mathrm{O}$ & $\mathrm{O}$ & \\
\hline 626 & Miscanthus sinensis Andersson for. purpurascens (Andersson) Nakai 억새 KP256215 & $\mathrm{O}$ & & & & $\mathrm{O}$ & $\mathrm{O}$ \\
\hline 627 & Moliniopsis japonica (Hack.) Hayata 진퍼리새 & & & & $\mathrm{O}$ & $\mathrm{O}$ & \\
\hline 628 & Muhlenbergia japonica Steud. 쥐꼬리새 KP256317 & & & & & & $\mathrm{O}$ \\
\hline 629 & Oplismenus undulatifolius (Ard.) Roem. \& Schult. 주름조개풀 KP291297 & $\mathrm{O}$ & & & & $\mathrm{O}$ & $\mathrm{O}$ \\
\hline
\end{tabular}


Appendix 1. Continued.

\begin{tabular}{|c|c|c|c|c|c|c|c|}
\hline \multirow{2}{*}{ No. } & \multirow{2}{*}{$\begin{array}{c}\text { Taxa and representative voucher specimen number } \\
\text { (KP: KOSPVP0000-, KN: KONPVP0000-, NP: NIBRVP0000-) }\end{array}$} & \multicolumn{6}{|c|}{ Study } \\
\hline & & 1 & 2 & 3 & 4 & 5 & 6 \\
\hline 630 & Panicum bisulcatum Thunb. 개기장 KP256250 & & & & $\mathrm{O}$ & & $\mathrm{O}$ \\
\hline 631 & Panicum dichotomiflorum Michx. 미국개기장 & & $\mathrm{O}$ & & $\mathrm{O}$ & & \\
\hline 632 & Parapholis incurva (L.) C. E. Hubb. 회초리잔디 KP318923 & & & & $\mathrm{O}$ & & $\mathrm{O}$ \\
\hline 633 & Paspalum distichum L. 물참새피 KP320402 & & $\mathrm{O}$ & & $\mathrm{O}$ & & $\mathrm{O}$ \\
\hline 634 & Paspalum distichum L. var. indutum Shinners 털물참새피 KP255645 & & $\mathrm{O}$ & & $\mathrm{O}$ & & $\mathrm{O}$ \\
\hline 635 & Paspalum thunbergii Kunth ex Steud. 참새피 KP319838 & & & & $\mathrm{O}$ & $\mathrm{O}$ & $\mathrm{O}$ \\
\hline 636 & Pennisetum alopecuroides (L.) Spreng. 수크령 KP256092 & & & & & & $\mathrm{O}$ \\
\hline 637 & Phacelurus latifolius (Steud.) Ohwi 모새달 & & & & $\mathrm{O}$ & & \\
\hline 638 & Phragmites communis Trin. 갈대 KP256196 & $\mathrm{O}$ & & & $\mathrm{O}$ & & $\mathrm{O}$ \\
\hline 639 & Phyllostachys bambusoides Siebold \& Zucc. 왕대 & & & & $\mathrm{O}$ & & \\
\hline 640 & Poa annua L. 새포아풀 KP318930 & & & & $\mathrm{O}$ & $\mathrm{O}$ & $\mathrm{O}$ \\
\hline 641 & Poa nipponica Koidz. 큰꾸러미풀 & & & & $\mathrm{O}$ & & \\
\hline 642 & Poa pratensis L. 왕포아풀 & & $\mathrm{O}$ & & $\mathrm{O}$ & $\mathrm{O}$ & \\
\hline 643 & Poa sphondylodes Trin. 포아풀 KP318905 & & & & $\mathrm{O}$ & $\mathrm{O}$ & $\mathrm{O}$ \\
\hline 644 & Polypogon fugax Nees ex Steud. 쇠돌피 KP256303 & & & & $\mathrm{O}$ & & $\mathrm{O}$ \\
\hline 645 & Polypogon monspeliensis (L.) Desf. 갯쇠돌피 KP318907 & & & $\mathrm{O}$ & $\mathrm{O}$ & $\mathrm{O}$ & $\mathrm{O}$ \\
\hline 646 & Pseudoraphis ukishiba Ohwi 물잔디 & & & & $\mathrm{O}$ & & \\
\hline 647 & Sacciolepis indica (L.) Chase 좀물뚝새 & & & & & $\mathrm{O}$ & \\
\hline 648 & Sacciolepis indica (L.) Chase 물뚝새 var. oryzetorum (Makino) Ohwi KP256266 & & & & $\mathrm{O}$ & & $\mathrm{O}$ \\
\hline 649 & Sasa borealis (Hack.) Makino \& Shibata 조릿대 & & & $\mathrm{O}$ & & $\mathrm{O}$ & \\
\hline 650 & Sasa japonica (Siebold \& Zucc. ex Steud.) Makino 이대 & & & & $\mathrm{O}$ & & \\
\hline 651 & Schizachyrium brevifolium (Sw.) Nees \& Büse 쇠풀 & & & & & $\mathrm{O}$ & \\
\hline 652 & Setaria $\times$ pycnocoma (Steud.) Henrard ex Nakai 수강아지풀 & & & & & $\mathrm{O}$ & \\
\hline 653 & Setaria faberi R. A. W. Herrm. 가을강아지풀 KP255717 & & & & $\mathrm{O}$ & & $\mathrm{O}$ \\
\hline 654 & Setaria glauca (L.) P. Beauv. 금강아지풀 KP256094 & & & & $\mathrm{O}$ & $\mathrm{O}$ & $\mathrm{O}$ \\
\hline 655 & Setaria viridis (L.) P. Beauv. 강아지풀 KP255690 & & & & & $\mathrm{O}$ & $\mathrm{O}$ \\
\hline 656 & $\begin{array}{l}\text { Setaria viridis (L.) P. Beauv. var. pachystachys (Franch. \& Sav.) Makino \& Nemoto 갯강아지풀 } \\
\text { KP319818 }\end{array}$ & & & & & & $\mathrm{O}$ \\
\hline 657 & Sorghum nitidum (Vahl) Pers. 수수새 KP256246 & & & & $\mathrm{O}$ & $\mathrm{O}$ & $\mathrm{O}$ \\
\hline 658 & Spodiopogon sibiricus Trin. 큰기름새 KP256334 & & & & & & $\mathrm{O}$ \\
\hline 659 & Sporobolus elongatus R. Br. 쥐꼬리새풀 KP256234 & $\mathrm{O}$ & & & $\mathrm{O}$ & $\mathrm{O}$ & $\mathrm{O}$ \\
\hline 660 & Themeda triandra Forssk. subsp. japonica (Willd.) T. Koyama 솔새 KP255743 & $\mathrm{O}$ & & & $\mathrm{O}$ & $\mathrm{O}$ & $\mathrm{O}$ \\
\hline 661 & Trisetum bifidum (Thunb.) Ohwi 잠자리피 & & & & $\mathrm{O}$ & & \\
\hline 662 & Zoysia japonica Steud. 잔디 & $\mathrm{O}$ & & & $\mathrm{O}$ & & \\
\hline 663 & Zoysia macrostachya Franch. \& Sav. 왕잔디 KP290416 & & & & & & $\mathrm{O}$ \\
\hline 664 & Zoysia sinica Hance 갯잔디 KP217360 & & & $\mathrm{O}$ & $\mathrm{O}$ & $\mathrm{O}$ & $\mathrm{O}$ \\
\hline & Typhaceae 부들과 & & & & & & \\
\hline 665 & Typha angustifolia L. 애기부들 KP255654 & & & & & & $\mathrm{O}$ \\
\hline 666 & Typha laxmannii Lepech. 꼬마부들 KP255655 & & & & & & $\mathrm{O}$ \\
\hline
\end{tabular}


Appendix 1. Continued.

\begin{tabular}{|c|c|c|c|c|c|c|c|}
\hline \multirow{2}{*}{ No. } & \multirow{2}{*}{$\begin{array}{c}\text { Taxa and representative voucher specimen number } \\
\text { (KP: KOSPVP0000-, KN: KONPVP0000-, NP: NIBRVP0000-) }\end{array}$} & \multicolumn{6}{|c|}{ Study } \\
\hline & & 1 & 2 & 3 & 4 & 5 & 6 \\
\hline \multicolumn{8}{|c|}{ Liliaceae 백합과 } \\
\hline 667 & Aletris spicata (Thunb.) Franch. 쥐꼬리풀 KP217366 & $\mathrm{O}$ & & $\mathrm{O}$ & $\mathrm{O}$ & $\mathrm{O}$ & $\mathrm{O}$ \\
\hline 668 & Allium macrostemon Bunge 산달래 KP255577 & & & & & & $\mathrm{O}$ \\
\hline 669 & Allium monanthum Maxim. 달래 & $\mathrm{O}$ & & & & & \\
\hline 670 & Allium sacculiferum Maxim. 참산부추 KP256243 & & & & & & $\mathrm{O}$ \\
\hline 671 & Allium thunbergii G. Don 산부추 KP256113 & & & & & $\mathrm{O}$ & $\mathrm{O}$ \\
\hline 672 & Disporum smilacinum A. Gray 애기나리 KP255440 & $\mathrm{O}$ & & & & $\mathrm{O}$ & $\mathrm{O}$ \\
\hline 673 & Hemerocallis fulva (L.) L. 원추리 & (a) & & & & & \\
\hline 674 & Hemerocallis minor Mill. 애기원추리 & & & & & $\mathrm{O}$ & \\
\hline 675 & Hemerocallis thunbergii Baker 노랑원추리 KP290423 & & & & $\mathrm{O}$ & & $\mathrm{O}$ \\
\hline 676 & Lilium amabile Palib. 털중나리 & & & & & $\mathrm{O}$ & \\
\hline 677 & Lilium tsingtauense Gilg 하늘말나리 KP255538 & & & & & $\mathrm{O}$ & $\mathrm{O}$ \\
\hline 678 & Liriope platyphylla F. T. Wang \& T. Tang 맥문동 KP289426 & & & & & $\mathrm{O}$ & $\mathrm{O}$ \\
\hline 679 & Polygonatum cryptanthum H. Lèv. \& Vaniot 목포용둥굴레 KP255539 & & & & $\mathrm{O}$ & & $\mathrm{O}$ \\
\hline 680 & Polygonatum falcatum A. Gray 진황정 & & & & & $\mathrm{O}$ & \\
\hline 681 & Polygonatum inflatum Kom. 퉁둥굴레 KP255452 & & & & & & $\mathrm{O}$ \\
\hline 682 & Polygonatum involucratum (Franch. \& Sav.) Maxim. 용둥굴레 KP255436 & $\mathrm{O}$ & & & & $\mathrm{O}$ & $\mathrm{O}$ \\
\hline 683 & Polygonatum lasianthum Maxim. 죽대 KP255537 & & & & & $\mathrm{O}$ & $\mathrm{O}$ \\
\hline 684 & Polygonatum odoratum (Mill.) Druce var. pluriflorum (Miq.) Ohwi 둥굴레 & $\mathrm{O}$ & & & & & \\
\hline \multirow[t]{2}{*}{685} & Scilla scilloides (Lindl.) Druce 무릇 KP255732 & $\mathrm{O}$ & & & $\mathrm{O}$ & & $\mathrm{O}$ \\
\hline & Iridaceae 붓꽃과 & & & & & & \\
\hline 686 & Iris ensata Thunb. var. spontanea (Makino) Nakai 꽃창포 & & & $\mathrm{O}$ & $\mathrm{O}$ & $\mathrm{O}$ & \\
\hline 687 & Iris pseudoacorus L. 노랑꽃창포 & & & & $\mathrm{O}$ & & \\
\hline 688 & Iris rossii Baker 각시붓꽃 $\mathrm{KP} 255372$ & $\mathrm{O}$ & & & & $\mathrm{O}$ & $\mathrm{O}$ \\
\hline 689 & Iris sanguinea Donn ex Hornem. 붓꽃 & $\mathrm{O}$ & & & & & \\
\hline \multirow[t]{2}{*}{690} & Sisyrinchium angustifolium Mill. 등심붓꽃 KP255597 & $\mathrm{O}$ & & & & & $\mathrm{O}$ \\
\hline & Smilacaceae 청미래덩굴과 & & & & & & \\
\hline 691 & Smilax china L. 청미래덩굴 KP255368 & $\mathrm{O}$ & & $\mathrm{O}$ & $\mathrm{O}$ & $\mathrm{O}$ & $\mathrm{O}$ \\
\hline \multirow[t]{2}{*}{692} & Smilax nipponica Miq. 선밀나물 KP255449 & & & & & $\mathrm{O}$ & $\mathrm{O}$ \\
\hline & Dioscoreaceae 마과 & & & & & & \\
\hline 693 & Dioscorea oppositifolia L. 마 KP255679 & & & & $\mathrm{O}$ & & $\mathrm{O}$ \\
\hline \multirow[t]{2}{*}{694} & Dioscorea quinqueloba Thunb. 단풍마 & $\mathrm{O}$ & & & & & \\
\hline & Orchidaceae 난초과 & & & & & & \\
\hline 695 & Bletilla striata (Thunb.) Rchb. f. 자란 KP217367 & $\mathrm{O}$ & & & $\mathrm{O}$ & & $\mathrm{O}$ \\
\hline 696 & Calanthe discolor Lindl. 새우난초 & & & & & $\mathrm{O}$ & \\
\hline 697 & Cephalanthera erecta (Thunb.) Blume 은난초 KP319546 & & & & & & $\mathrm{O}$ \\
\hline 698 & Cephalanthera falcata (Thunb.) Blume 금난초 & & & & & $\mathrm{O}$ & \\
\hline 699 & Cymbidium goeringii (Rchb. f.) Rchb. f. 보춘화 KP255429 & $\mathrm{O}$ & & & $\mathrm{O}$ & & $\mathrm{O}$ \\
\hline 700 & Epipactis thunbergii A. Gray 닭의난초 KP255755 & $\mathrm{O}$ & & & & $\mathrm{O}$ & $\mathrm{O}$ \\
\hline
\end{tabular}


Appendix 1. Continued.

\begin{tabular}{|c|c|c|c|c|c|c|c|}
\hline \multirow{2}{*}{ No. } & \multirow{2}{*}{$\begin{array}{l}\text { Taxa and representative voucher specimen number } \\
\text { (KP: KOSPVP0000-, KN: KONPVP0000-, NP: NIBRVP0000-) }\end{array}$} & \multicolumn{6}{|c|}{ Study } \\
\hline & & 1 & 2 & 3 & 4 & 5 & 6 \\
\hline 701 & Habenaria linearifolia Maxim. 잠자리난초 KP255769 & & & & $\mathrm{O}$ & $\mathrm{O}$ & $\mathrm{O}$ \\
\hline & Platanthera mandarinorum Rchb. f. 산제비란 & & & & $\mathrm{O}$ & & \\
\hline & Pogonia japonica Rchb. f. 큰방울새란 KP255526 & & & & $\mathrm{O}$ & & $\mathrm{O}$ \\
\hline & Pogonia minor (Makino) Makino 방울새란 & & & & $\mathrm{O}$ & & \\
\hline 705 & Spiranthes sinensis (Pers.) Ames 타래난초 KP255563 & $\mathrm{O}$ & & & $\mathrm{O}$ & & $\mathrm{O}$ \\
\hline
\end{tabular}

1, Park (2004); 2, Kim (2008); 3, Jeong et al. (2011); 4, Nam et al. (2012); 5, Hwang et al. (2013); 6, This study; @, cultivated plants. 